\title{
Lung CD103+ dendritic cells restrain allergic airway inflammation through IL-12 production
}

\author{
Laura Conejero, Sofía C. Khouili, Sarai Martínez-Cano, Helena M. Izquierdo, Paola Brandi, \\ and David Sancho \\ Centro Nacional de Investigaciones Cardiovasculares Carlos III (CNIC), Melchor Fernández Almagro 3, Madrid, Spain.
}

\begin{abstract}
$D C s$ are necessary and sufficient for induction of allergic airway inflammation. CD11b+ DCs direct the underlying Th2 immunity, but debate surrounds the function of $\mathrm{CD}_{103^{+}} \mathrm{DCs}$ in lung immunity and asthma after an allergic challenge. We challenged Batf $3^{-/-}$mice, which lacked lung $C D 103^{+} D C s$, with the relevant allergen house dust mite (HDM) as a model to ascertain their role in asthma. We show that acute and chronic HDM exposure leads to defective Th1 immunity in Batf3-deficient mice. In addition, chronic HDM challenge in Batf3 $3^{-/-}$mice results in increased Th2 and Th17 immune responses and exacerbated airway inflammation. Mechanistically, Batf3 absence does not affect induction of Treg or IL-10 production by lung CD4+ $\mathrm{T}$ cells following acute HDM challenge. Batf3dependent $\mathrm{CD}_{103}{ }^{+}$migratory DCs are the main source of IL-12p40 in the mediastinal lymph node DC compartment in the steady state. Moreover, $\mathrm{CD} 103^{+} \mathrm{DCs}$ selectively increase their IL-12p40 production upon HDM administration. In vivo IL-12 treatment reverts exacerbated allergic airway inflammation upon chronic HDM challenge in Batf3--- mice, restraining Th2 and Th17 responses without triggering Th1 immunity. These results suggest a protective role for lung CD103+ DCs to HDM allergic airway inflammation through the production of IL-12.
\end{abstract}

Conflict of interest: The authors have declared that no conflict of interest exists.

Submitted: August 30, 2016

Accepted: April 7, 2017

Published: May 18, 2017

\section{Reference information:}

JCI Insight. 2017;2(10):e90420. https://doi.org/10.1172/jici. insight.90420.

\section{Introduction}

Asthma is a chronic inflammatory disease that identifies a wide spectrum of respiratory-related symptoms, typically associated to eosinophilia, with variable and often reversible airway obstruction accompanied by airway hyperreactivity (AHR). Asthma is a major cause of disability and the most common chronic disease among children and young adults. DCs play a pivotal role in the immune response to inhaled allergens by taking them up, transporting them to the draining mediastinal lymph nodes $(\mathrm{mLN})$, and presenting antigens to initiate the $\mathrm{CD} 4^{+} \mathrm{Th}$ response (1). The main DC subsets in the steady state in the lung are plasmacytoid DCs and conventional DCs, the latter subdivided into 2 functionally distinct subsets that have been recently renamed as classical type 1 DCs (cDC1, Batf3-dependent) and classical type 2 DCs (cDC2, IRF4-dependent) and that are defined by surface marker expression and development in the airways (2-5). Attending to their migratory properties, cDCs are lymphoid organ-resident DCs (res-DCs) $\left(\mathrm{CD} 8 \alpha^{+}, \mathrm{CD} 24^{+}\right.$, and XCR1 $1^{+}$ $\mathrm{cDC} 1 \mathrm{~s}$ and $\mathrm{CD} 11 \mathrm{~b}^{+} \mathrm{cDC} 2 \mathrm{~s}$ ) and tissue-derived or migratory DCs (mig-DCs) consisting of $\mathrm{CD} 103^{+} \mathrm{XCR} 1^{+}$ $\mathrm{cDC} 1 \mathrm{~s}$ and $\mathrm{CD} 11 \mathrm{~b}^{+} \mathrm{SIRP} \alpha^{+} \mathrm{CDC} 2 \mathrm{~s}$, present in the lung and trafficking to the mLNs $(6,7)$. Upon lung inflammation, the lungs are also infiltrated by monocyte-derived DCs (1). Much effort in the field is currently directed at elucidating the division of labor among different DC subsets in the context of asthma, and contributions by several DC subsets have been defined. It was suggested that mouse lung cDC1s predominantly prime Th1 and Th17 responses in vitro, whereas mouse lung cDC2s foster a Th2 response (8). Plasmacytoid DCs play a key role in the induction of Tregs that control exacerbated airway inflammation (9). CD11b cDC2s mediate Th2 priming in response to house dust mite (HDM) and to Blomia tropicalis mites (10-12), leading to eosinophilic airway inflammation, mucus production, and AHR. CD11 b $b^{+} \mathrm{cDC} 2 \mathrm{~s}$ also promote a Th17 response upon lung fungal infection with Aspergillus fumigatus (13). Th17 immunity contributes to neutrophilic inflammation associated with some severe forms of asthma (14). Monocyte-derived DCs play a major proinflammatory function, contributing significantly to the immunopathology of asthma through the production of chemokines (10). However, debate still surrounds the function of $\mathrm{CD}_{103}{ }^{+} \mathrm{CDC} 1 \mathrm{~s}$ in Th2 induction and the development of allergic asthma (10, 15-17). 
The development and function of some DC subsets is mediated by the basic leucine zipper transcription factor ATF-like 3 (Batf3) (18-20). In the C57BL/6 background, the main subset affected in tissues and draining $\mathrm{LNs}$ is $\mathrm{CD}_{103}{ }^{+} \mathrm{cDC} 1 \mathrm{~s}$, while functionally and developmentally related $\mathrm{CD} 8 \alpha^{+}$res-cDC1s are partially impaired in the LNs (18-20). Batf3-dependent lung CD103 ${ }^{+} \mathrm{cDC} 1 \mathrm{~s}$ are required for transport of influenza virus to the mLNs, for the generation of $\mathrm{CD} 8^{+} \mathrm{T}$ cell immunity against influenza or Sendai virus $(19,21,22)$, and for priming of resident memory $\mathrm{CD} 8^{+} \mathrm{T}$ cells against vaccinia virus (23). Moreover, Aspergillus-germinated morphotypes induce IL-2 in lung CD $103^{+} \mathrm{DCs}$, leading to an optimal Th17 response (24). Here, we explore the debated role of CD103+ DCs in HDM-induced allergic asthma. We report that Batf3-deficient mice develop exacerbated Th2- and Th17-driven allergic asthma when chronically exposed to HDM. CD103+ DCs are key regulators that control Th2 and Th17 responses in allergen-exposed mice not by inducing Tregs, but rather by increasing IL-12p40 production.

\section{Results}

Batf $3^{-1-}$ mice show severely impaired $C D 103^{+}$cDC1 compartment. To evaluate the role of Batf3-dependent $\mathrm{cDC} 1 \mathrm{~s}$ during asthma, we analyzed Batf3-deficient mice after sensitization and challenge with HDM. We first assessed Batf3-dependent $\mathrm{cDC} 1 \mathrm{~s}$ in both the mLN and lung, since those are the tissues involved in the initiation and the effector phase, respectively, in asthma. To analyze DCs in the lung, WT and Batf $3^{-1-}$ mice were sensitized (i.n.) to HDM on day $0(1 \mu \mathrm{g})$ and then challenged i.n. on days 7-11 with $10 \mu \mathrm{g}$ HDM (Figure 1A and Supplemental Figure 1A; supplemental material available online with this article; https://doi.org/10.1172/jci.insight.90420DS1) (10, 25). When defining our gating strategy for DCs, we first gated out alveolar macrophages based on their CD11c and Siglec-F expression and their strong autofluorescence (10) (Figure 1B and Supplemental Figure 1B). The lungs of WT mice had a reduced frequency of $\mathrm{CD} 103^{+} \mathrm{XCR} 1^{+} \mathrm{SIRP} \alpha \mathrm{CD} 24^{\text {hi }} \mathrm{CD} 11 \mathrm{~b}^{\text {lo }} \mathrm{CDC} 1 \mathrm{~s}$ in the $\mathrm{CD} 11 \mathrm{c}^{+} \mathrm{MHCII}^{+}$compartment on day 14 following HDM sensitization and challenge (Figure 1C and Supplemental Figure 1C), probably due to the recruitment of CD11b+ $\mathrm{cDC} 2 \mathrm{~s}$ and monocyte-derived DCs (moDCs) to the lung (10). Batf3 ${ }^{-1-}$ mice exposed to PBS lacked $\mathrm{CD} 103^{+} \mathrm{XCR} 1^{+} \mathrm{cDC} 1 \mathrm{~s}$, a subset that was not restored following HDM challenge.

For the assessment of early DC response in the $\mathrm{mLN}$ to allergen challenge, mice were exposed to $100 \mu \mathrm{g} \mathrm{HDM}$ (Figure 1D and Supplemental Figure 1D), which is the dose administered in previous studies where HDM uptake and migration by pulmonary myeloid DCs was assessed $(10,26)$. WT mice exposed to a single HDM dose had a decreased percentage of CD8 $\alpha^{+}$res-cDC1s and CD103+ mig-cDC1s in the DC compartment in the mLNs (Figure 1, E-G), but the total number was not decreased on day 3 after challenge compared with PBS controls (data not shown). Batf3 ${ }^{-1-}$ mice lacked $\mathrm{CD}_{103}{ }^{+} \mathrm{XCR} 1^{+} \mathrm{SIRP} \alpha \mathrm{CD} 24^{\mathrm{hi}} \mathrm{CD} 11 \mathrm{~b}^{\text {lo }} \mathrm{CDC} 1 \mathrm{~s}$ in the $\mathrm{mLNs}$ and showed reduced res-CD $8 \alpha^{+} \mathrm{CDC} 1$ in the mLNs (Figure 1, E-G, and Supplemental Figure 1, E-G), the remaining res-CD $8 \alpha^{+} \mathrm{cDC} 1$ being XCR $1^{-}$ (27). The similar DC profile in the $\mathrm{mLN}$ of $\mathrm{Batf}^{-{ }^{--}}$mice, regardless of HDM challenge, suggests that the treatment does not restore $\mathrm{CD} 103^{+} \mathrm{DCs}$ in the lungs of Batf3-1- mice, in contrast with the effect of aerosol exposure to Mycobacterium tuberculosis (28).

Impaired Th1 immunity in Batf $3^{-1-}$ mice upon acute HDM challenge. Next, WT and Batf3 ${ }^{-1-}$ mice were sensitized i.n. to HDM on day $0(1 \mu \mathrm{g})$ and challenged i.n. for 5 consecutive days (on days 7-11, $10 \mu \mathrm{g}$ HDM) (25), and different hallmarks of asthma were evaluated 3 days after the last challenge. Analysis of bronchoalveolar lavage (BAL) revealed similar inflammatory infiltration in Batf3-deficient and WT mice at day 14 (Figure 2A). Moreover, histological analysis of lung sections showed similarly increased perivascular and peribronchial inflammation and mucus production (Figure 2B). However, while serum levels of HDMspecific IgG1 were similar in Batf3 ${ }^{-/-}$and WT mice (Figure 2C), HDM-exposed Batf3 $3^{-/}$mice did not induce the production of HDM-specific IgG2a (Figure 2D), an antibody isotype associated with Th1 immunity.

To further dissect the specificity of the generated immune response, we restimulated mLNs and splenocytes from day $14 \mathrm{HDM}$-challenged WT and Batf3 ${ }^{-/-}$mice with HDM extract. Tissue from Batf3-deficient mice showed a selective impairment in IFN- $\gamma$ production, while production of IL-5, IL-17A, and IL-10 was similar locally (mLNs) and systemically (splenocytes) (Figure 3). We additionally restimulated lung

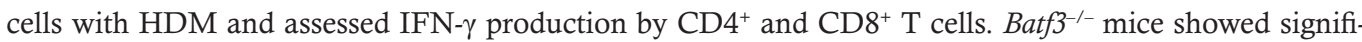
cantly lower production of IFN- $\gamma$ by CD4 ${ }^{+} \mathrm{T}$ cells compared with WT controls (Supplemental Figure 2A). Notably, we did not detect IFN- $\gamma$ production by $\mathrm{CD} 8^{+} \mathrm{T}$ cells in response to HDM challenge in any of the experimental groups (Supplemental Figure 2B). These results suggest that Batf3-dependent DCs are essential for induction of $\mathrm{CD} 4^{+} \mathrm{Th} 1$ immunity after acute HDM challenge. 


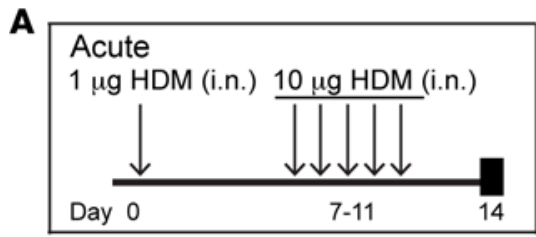

B Lung, gated on $\mathrm{CD}_{4} 5^{+}$cells

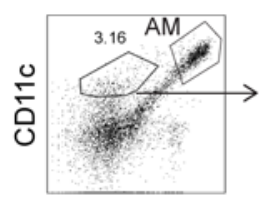

Siglec F

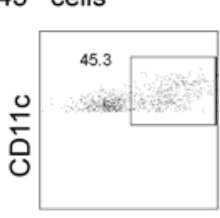

$\mathrm{MHCII}$
C

Lung, gated on $\mathrm{CD}^{+} 5^{+}$
Siglec $\mathrm{F}^{-} \mathrm{MHCII}^{+}$cells

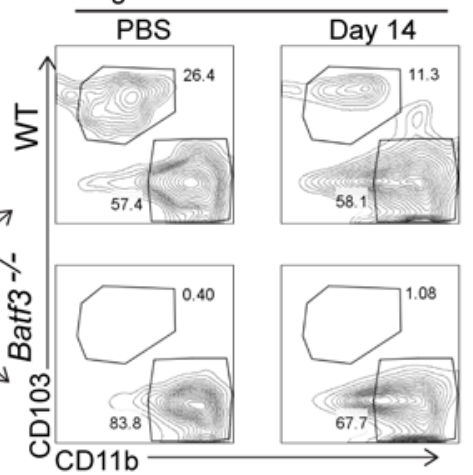

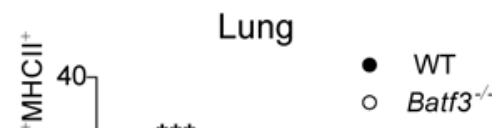

$\begin{array}{ll}- & \text { WT } \\ \circ & \text { Batf3 }^{-1}\end{array}$
D

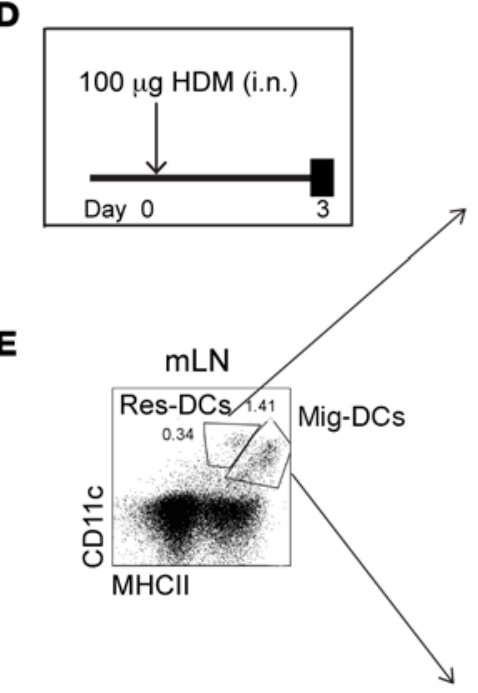

$\mathbf{F}$

Res-DCs (Gated on CD11c ${ }^{+} \mathrm{MHCII}^{\text {mid }}$ )
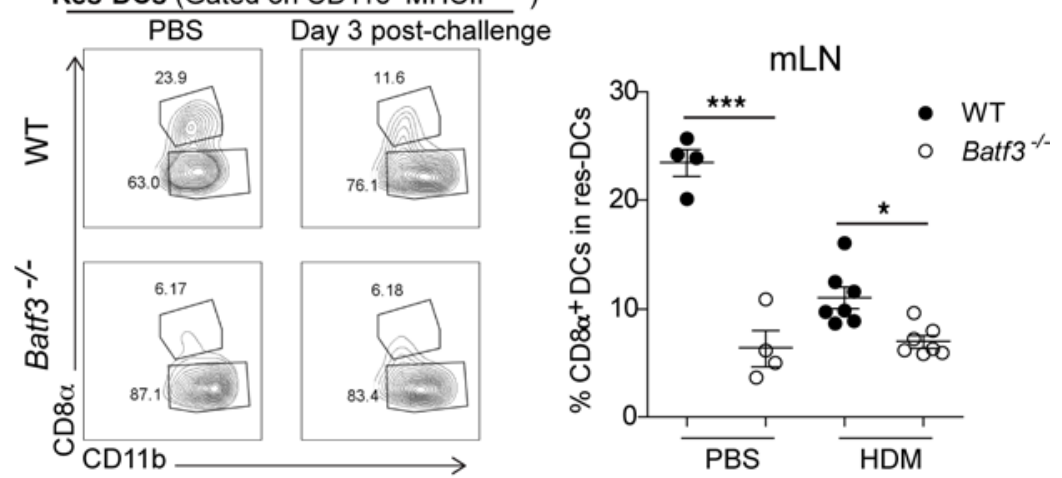

G

Mig-DCs (Gated on CD11c ${ }^{+} \mathrm{MHCII}^{\mathrm{hi}}$ )
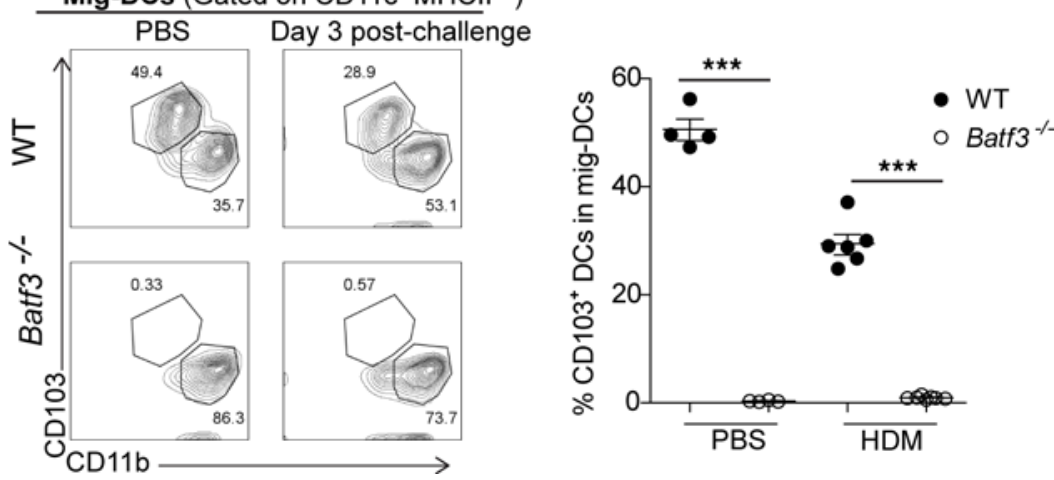

Figure 1. Batf3 ${ }^{-/-}$mice lack $C D 103^{+}$CDC1s in the lung and $\mathrm{mLN}$ and show a reduced $\mathrm{CD} 8 \boldsymbol{\alpha}^{+} \mathrm{CDC1}$ compartment in mLN. (A) HDM sensitization and challenge regime (acute protocol). (B) Gating strategy for lung Batf3-dependent DCs. (C) Staining of lung CD103 ${ }^{+}$and CD11 b+ DCs in mice treated as indicated in $\mathbf{A}$. Left: representative plots. Right: percentage of $\mathrm{CD} 103^{+} \mathrm{CDC1s}$ in the $\mathrm{CD}_{11 \mathrm{c}^{+} \mathrm{MHCII}}{ }^{+}$compartment in the lung. (D) $\mathrm{HDM}$ administration protocol to evaluate early DC response following allergen challenge. (E) Gating strategy to identify migratory (mig) and resident (res) DCs in mLNs. (F and $\mathbf{G})$ Res-DCs (F) and mig-DCs (G) in mLNs from mice i.n. challenged with $100 \mu \mathrm{g} \mathrm{HDM}$. Left: representative plots. Right: percentage of Batf3-dependent DCs in the CD11 $\mathrm{c}^{+} \mathrm{MHCII}{ }^{+}$ compartment in the $\mathrm{mLN}$. (C, F, and $\mathbf{G})$ Individual data $(n=3-4 \mathrm{PBS}, n=6-7 \mathrm{HDM})$ and mean \pm SEM of 1 representative experiment of 3 performed. ${ }^{*} P<$ $0.05,{ }^{* *} P<0.001,1$-way ANOVA followed by Bonferroni's post-test. HDM, house dust mite; mLN, mediastinal lymph nodes.

Lack of Batf3 exacerbates the Th2 and Th17 response to chronic HDM airway exposure. Although asthma has classically been associated with eosinophilia and Th2 cytokines, some patients show a neutrophilpredominant disease with an absence or low Th2 cytokine profile (14). Eosinophilia usually predominates in acute inflammation, while neutrophilia and IL-17 is common in chronic disease (29). Despite the lack of Th1 immunity in Batf $3^{-/}$mice upon acute HDM challenge, we did not find differences in the typical hallmarks of asthma (Figure 2 and Figure 3). Therefore, we next investigated the role of Batf3-dependent 
A

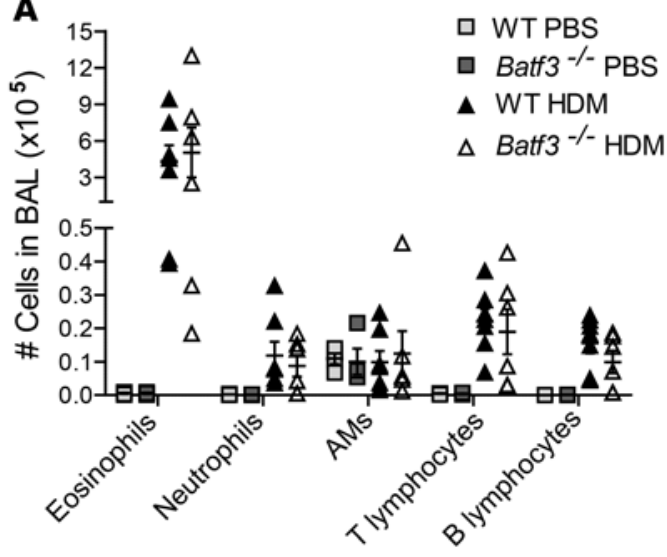

B
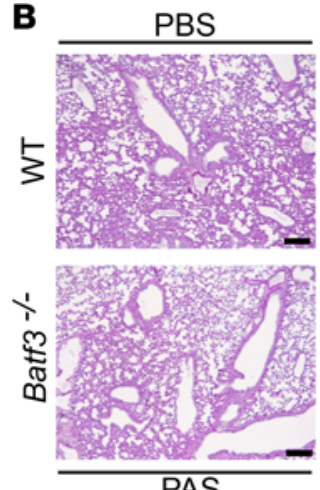

PAS

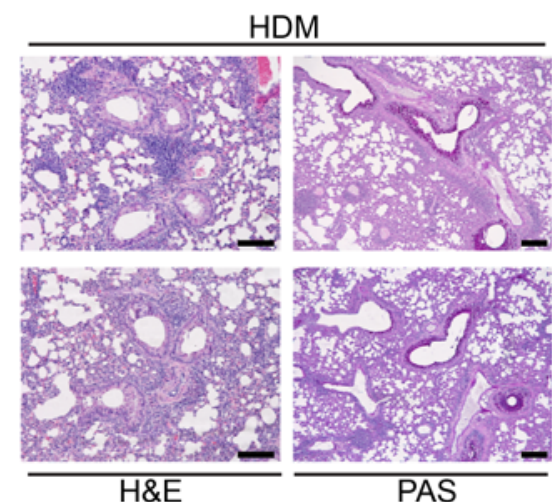

- WT
C

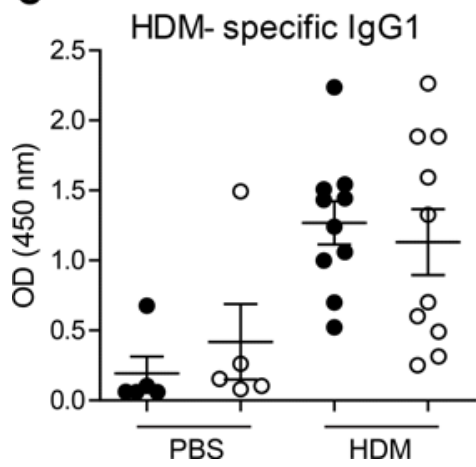

D

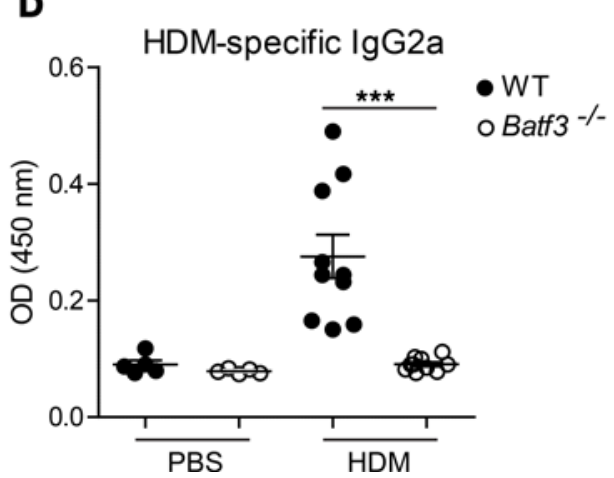

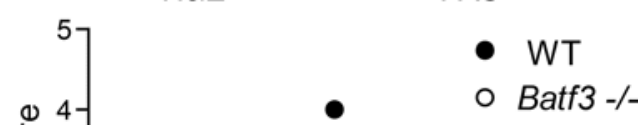

$\underline{0000}$

○

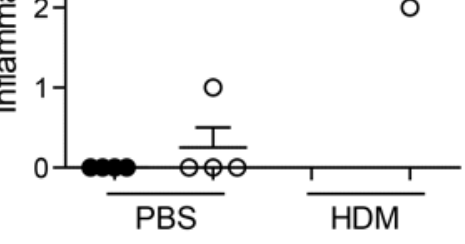

Figure 2. Batf3 deficiency does not impact on airway inflammation following acute HDM exposure. (A) Inflammatory infiltrates in BAL of HDM-sensitized and -challenged Batf3 ${ }^{-/}$and WT mice ( $n=3$ PBS, $n=7$ HDM). (B) Top: representative H\&E and PAS staining in formalin-fixed lung sections. Scale bars: $200 \mu \mathrm{m}$ (PAS) or $100 \mu \mathrm{m}$ (H\&E). Bottom: inflammation and mucus score ( $n=4$ PBS, $n=6$ HDM). (C and D) Serum HDM-specific IgG1 (C) and IgG2a (D) determined by ELISA ( $n=5$ PBS, $n=10$ HDM). (A-D) Individual data and mean \pm SEM of 1 representative experiment of at least 3 performed. ${ }^{* * *} P<0.001$, Mann Whitney $U$ test. HDM, house dust mite; PAS, periodic acid Schiff.

DCs in a model that recapitulates clinical features of severe chronic asthma, including neutrophilia (14). We postulated that lack of Th1 immunity in $\mathrm{Batf}^{-1-}$ mice would have a detrimental effect on HDM airway exposure over the long-term, aggravating the asthmatic phenotype by disturbing the Th1/Th2/Th17 balance. After chronic airway exposure to $\operatorname{HDM}(3 \times 10 \mu \mathrm{g}$ doses per week for 6 weeks), eosinophil and neutrophil influx was dramatically increased in Batf3 $3^{-/}$mice compared with WT mice (Figure 4B). Histological analysis revealed enhanced inflammation, goblet cell metaplasia, mucus production, and airway collagen deposition in HDM-treated mice (Figure 4C). HDM-exposed Batf3-/- mice showed significantly increased perivascular and peribronchiolar inflammation (Figure 4C). Total serum IgE, a marker of allergic sensitization, was increased in Batf3-deficient mice (Figure 4D). Consistent with the data from the acute model, Th1-linked HDM-specific IgG2a production was impaired in chronically HDM-exposed Batf3-1mice compared with similarly treated WT mice (Figure 4E). However, Batf3 $3^{--}$and WT mice showed no differences in HDM-specific IgG1 titers after chronic HDM challenge (Figure 4F).

Restimulation of mLNs and splenocytes isolated from chronically HDM-exposed mice revealed exacerbated local and systemic IL-5 and IL-17A production in Batf $3^{-1-}$ mice (Figure 5, A, B, E, and F). There were no differences in IL-10 production (Figure 5, C and G). Consistent with the data from the acute model, IFN- $\gamma$ production was impaired in Batf3-deficient mice (Figure 5, D and H). These data suggest that Batf3-dependent DCs, through their contribution to Th1 immunity, might restrain the allergic response and airway inflammation triggered by chronic HDM challenge.

Treg response after HDM challenge is not affected in the absence of Batf3. Previous evidence indicates that Batf3-dependent DCs in the lung contribute to airway homeostasis by inducing Tregs (16), which can suppress Th2 cell-mediated allergic reactions (30). Although IL-10 production did not differ between HDMrestimulated $\mathrm{Batf}^{-/-}$and WT tissues from acutely or chronically exposed mice (Figure 3, C and G, and 

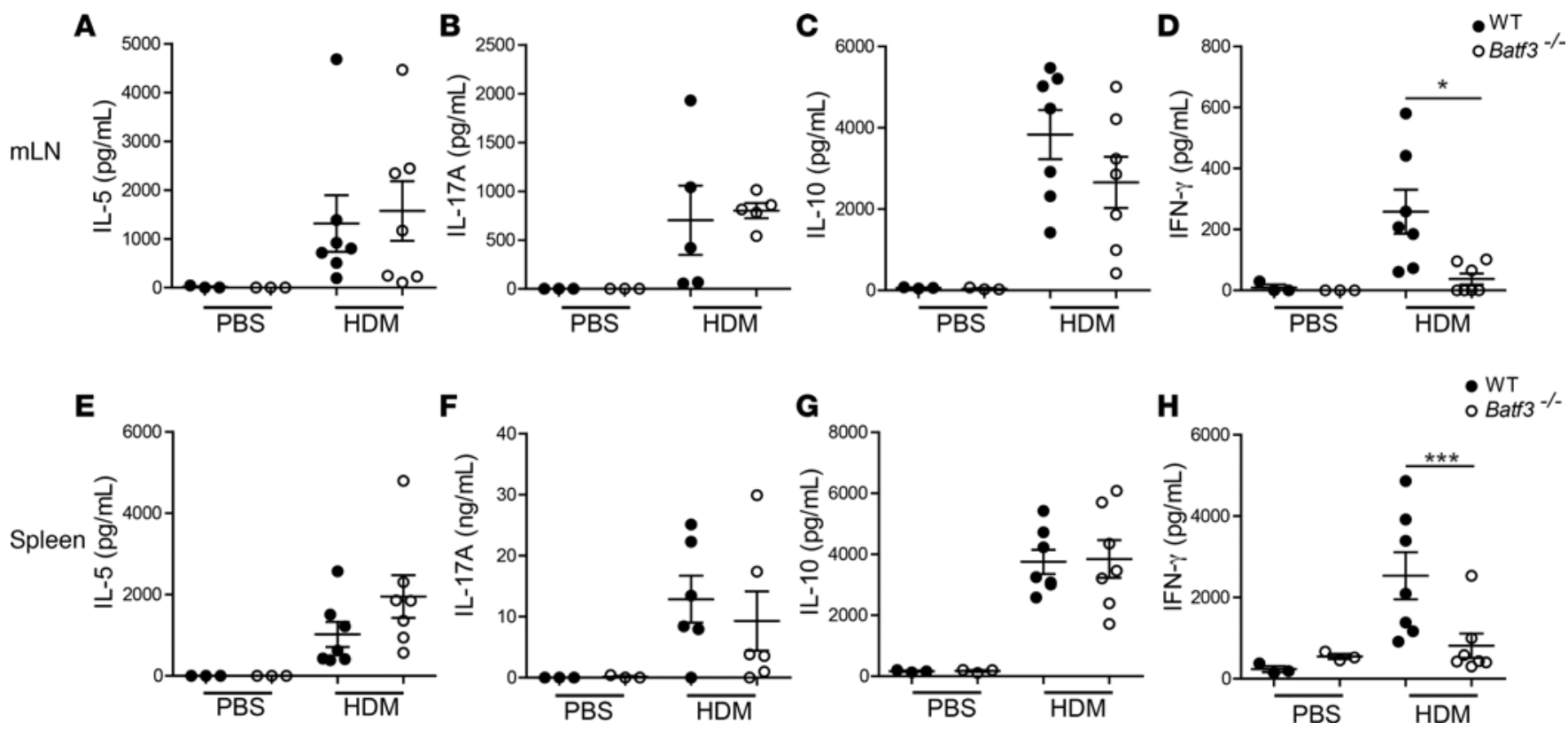

Figure 3. Batf3 ${ }^{-/-}$mice show impaired local and systemic IFN- $\gamma$ production upon acute HDM challenge. mLNs (A-D) and splenocytes (E-H) from HDMsensitized and HDM-challenged mice were restimulated with HDM, and released cytokines were measured by ELISA. Individual data and mean \pm SEM from a representative experiment of at least 3 performed ( $n=3$ PBS, $n=7 \mathrm{HDM}$ ); ${ }^{*} P<0.05,{ }^{* *} P<0.001$, Mann Whitney $U$ test. HDM, house dust mite; $\mathrm{mLN}$, mediastinal lymph node.

Figure 5, C and G), we analyzed whether Batf3-dependent DCs promote Treg expansion after allergen exposure. HDM sensitization and acute challenge increased Treg frequencies and total numbers in the lung (FoxP3 ${ }^{+} \mathrm{CD} 25^{+} \mathrm{CD}^{+} \mathrm{T}$ cells) to the same extent in WT and Batf3-deficient mice (Figure 6A). HDM exposure, thus, promotes FoxP3 ${ }^{+}$Treg differentiation, regardless of the presence of Batf3-dependent DCs.

The induction of adaptive FoxP3 ${ }^{+}$Treg cells is essential for establishing mucosal tolerance via the respiratory and oral routes, and these cells modulate sensitization to allergens and the severity of chronic inflammation (31). Inducible Tregs (iTregs, ICOS ${ }^{+}$) differentiate in the periphery as a consequence of antigen exposure (32), whereas natural Tregs (nTregs, Helios ${ }^{+}$) develop in the thymus (33). Analysis of FoxP3 ${ }^{+} \mathrm{CD}^{+} \mathrm{T}$ cells from the lungs of HDM-sensitized and -challenged mice showed a drop in the frequency of Helios ${ }^{+}$nTregs and an increased frequency of ICOS ${ }^{+}$iTregs; however, these changes were similar in WT and Batf $3^{-1-}$ mice, indicating that Batf3 deficiency does not selectively affect iTreg frequency after HDM challenge (Figure 6, B and C).

Although Batf3 deficiency appears not to affect Treg frequencies and numbers, it could affect Treg function. We therefore analyzed $\mathrm{CD} 4^{+}$effector $\mathrm{T}$ cells $\left(\mathrm{CD} 44^{+} \mathrm{T}\right.$ cells) in the lung for the production of the antiinflammatory cytokine IL-10, an essential immune regulator in mucosal tissues, such as lung and intestine (34). HDM exposure increased IL-10 production by CD44 ${ }^{+} \mathrm{T}$ cells (Figure $6 \mathrm{D}$ ), but the absence of Batf3 did not impair this effect. Together, these data indicate that Batf3-dependent DCs are neither required for Treg expansion nor involved in IL-10 production by effector $\mathrm{CD} 4^{+} \mathrm{T}$ cells after airway exposure to HDM.

$C D 103^{+}$mig-cDC1s are the main DC source of IL-12 in the mLNs upon HDM exposure. The impaired HDMinduced Th1 immunity in the absence of Batf3 prompted us to investigate whether HDM induces IL-12 secretion by Batf3-dependent lung $\mathrm{CD} 103^{+} \mathrm{CDC} 1 \mathrm{~s}$; these DCs would migrate to the draining $\mathrm{mLNs}$ to produce IL-12 that promotes Th1 differentiation, providing a mechanism for dampening the Th2 and Th17 immune response $(35,36)$. Mice were exposed to a single $100 \mu \mathrm{g} \mathrm{HDM}$ dose, and 3 days later, $\mathrm{CD} 11 \mathrm{c}^{+} \mathrm{DCs}$ purified from Batf $3^{-/-}$mLNs showed impaired IL-12p40 mRNA expression (Figure 7A). We also examined mRNA expression of IL- 6 , a cytokine produced by $\mathrm{CD} 103^{+} \mathrm{DCs}$ in the intestine (37) and by airway $\mathrm{CD} 11 \mathrm{~b}^{+} \mathrm{DCs}(13)$. The CD11 $\mathrm{c}^{+}$fraction in mLNs showed no difference in IL-6 mRNA expression between WT and Batf $3^{-1-}$ mice (Figure 7B).

Intracellular staining in mLN mig-DCs isolated after HDM challenge confirmed enhanced IL-12p40 production in WT compared with Batf $3^{-1-}$ mice (Figure 7C). Among the mig-DC subsets, comprising CD11 $\mathrm{b}^{+}$ cDC2s and Batf3-dependent $\mathrm{CD} 103^{+} \mathrm{CDC} 1 \mathrm{~s}, \mathrm{CD} 103^{+} \mathrm{CDC} 1 \mathrm{~s}$ were the main contributors to IL-12p40 produc- 
A
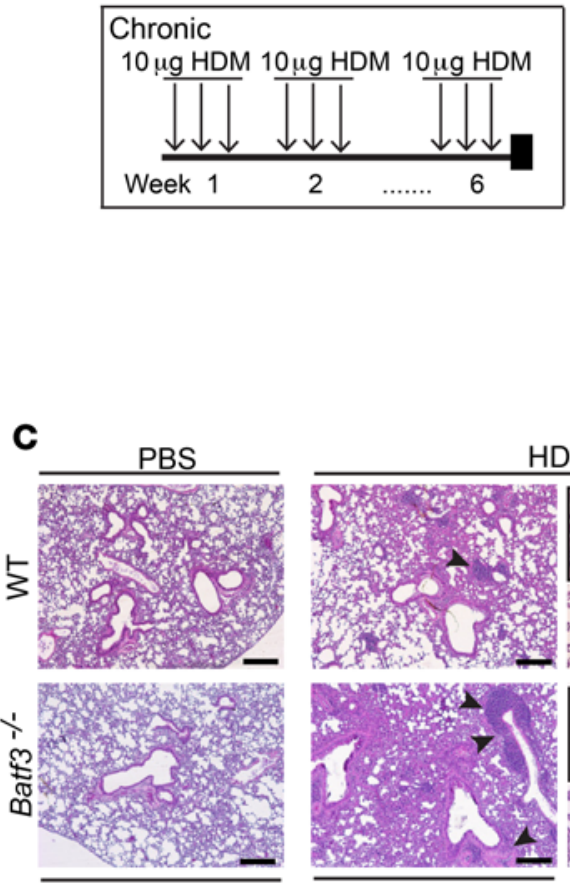

PAS

PBS

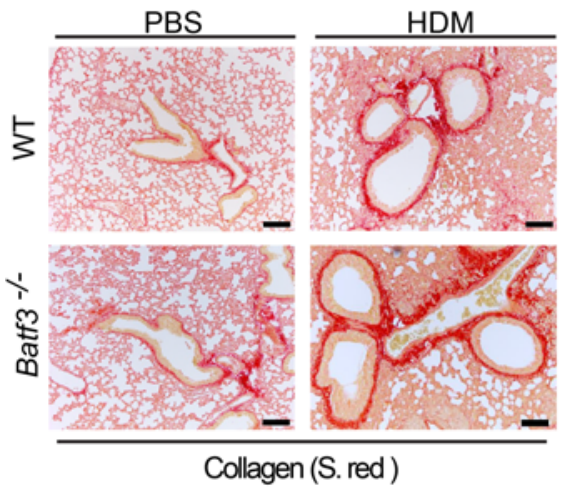

E

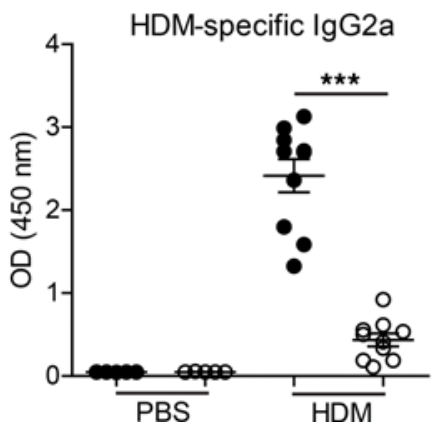

B

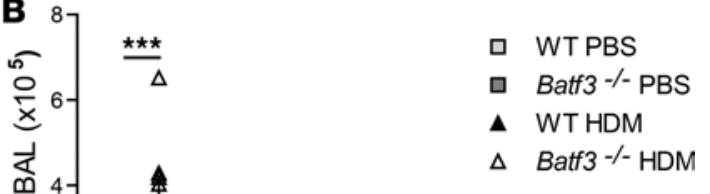

$\triangle$ Batf3 -/- HDM

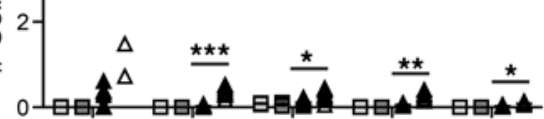

$5^{2} x^{x^{2}}$

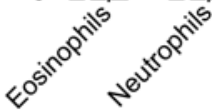

HDM

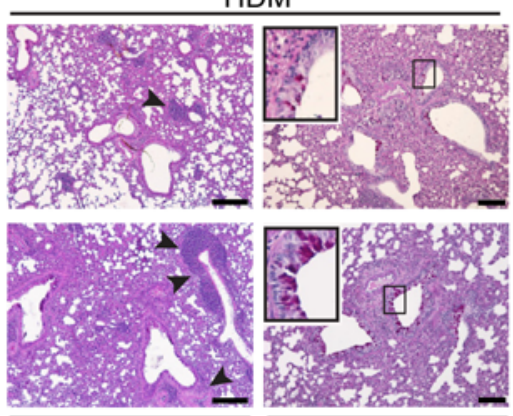

PAS

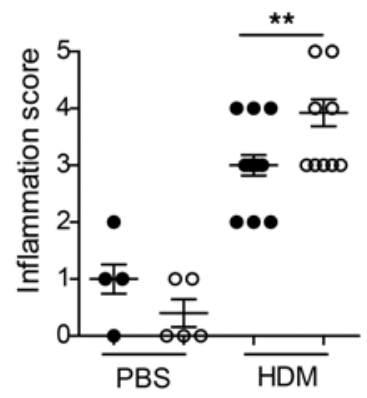

D
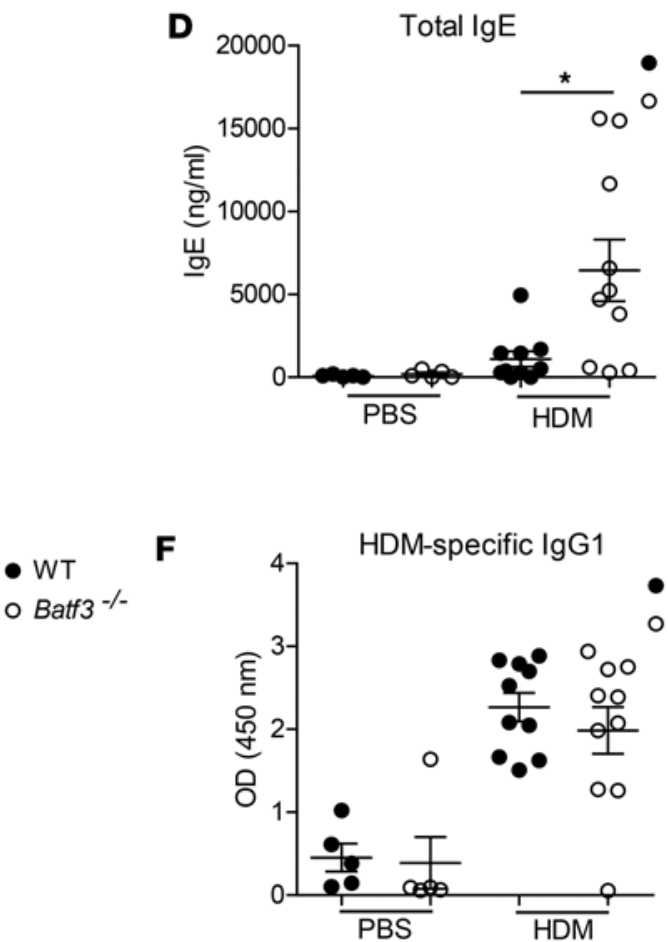

F

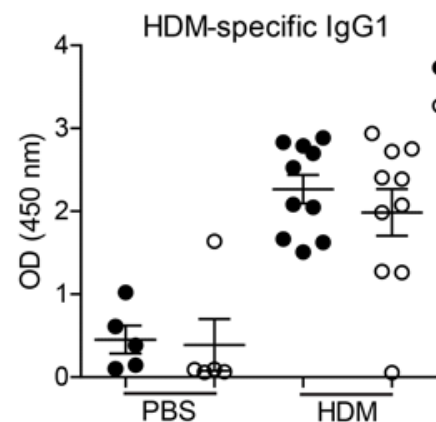

Figure 4. Batf3 $3^{-/-}$mice develop exacerbated HDM-induced chronic airway inflammation. (A) Schematic regime of chronic HDM exposure. (B) Inflammatory infiltrates in BAL from the indicated treatments and genotypes $(n=$ 3 PBS, $n=7$ HDM). (C) Representative histological staining of mice of the indicated genotype chronically exposed to HDM. Top: H\&E staining (arrowheads point at perivascular and peribronchial infiltration) and PAS staining (insets depict mucus-producing goblet cells). Bottom: staining for collagen (Sirius red). Scale bars: $200 \mu \mathrm{m}$ (PAS-PBS or H\&E) or $100 \mu \mathrm{m}$ (PAS-HDM or Sirius red). Right: inflammation score $(n=5$ PBS, 9 HDM). (D-F) Total serum IgE (D) and HDM-specific IgG2a (E) and IgG1 (F) ( $n=5$ PBS, $n=10$ HDM). (B-F) Individual data and mean \pm SEM from 1 experiment representative of at least 3 independent experiments; ${ }^{*} P<0.05$, ${ }^{*} P<0.01,{ }^{* *} P<0.001$, Mann Whitney $U$ test. HDM, house dust mite; PAS, periodic acid Schiff.

tion, in terms of both frequency and numbers, and both before and after HDM challenge (Figure 7, D and E). Significantly increased production of IL$12 \mathrm{p} 40$ by $\mathrm{CD} 103^{+} \mathrm{cDC} 1 \mathrm{~s}$ was also found when mice were exposed to a single $10 \mu \mathrm{g} \mathrm{HDM}$ i.n. dose (Supplemental Figure $3 \mathrm{~A}) . \mathrm{CD} 1 \mathrm{~b}^{+}$mig-DCs were unable to compensate for the impaired IL-12p40 production in Batf3-deficient mice. Notably, CD8 $\alpha^{+}$res-cDC1s did not produce any IL-12p40 following HDM i.n. challenge in WT or Batf $3^{--}$mice (Supplemental Figure 3, B and C). These results indicate that $\mathrm{CD}_{103}{ }^{+}$ $\mathrm{cDC} 1 \mathrm{~s}$ are key providers of IL-12 in the DC compartment upon HDM challenge.

Exogenous IL-12 is sufficient to suppress exacerbated allergic inflammation. To evaluate whether IL-12 delivery is the essential signal provided by $\mathrm{CD} 103^{+} \mathrm{CDC} 1 \mathrm{~s}$ to restrain an exacerbated $\mathrm{Th} 2$ and $\mathrm{Th} 17$ response, we administered IL-12 i.n. to Batf $3^{-1-}$ mice chronically exposed to HDM. Exogenous IL-12 provision was sufficient to drastically reduce BAL cellularity, mainly eosinophilia, to WT levels (Figure 8A). Similarly, IL-12 was sufficient to decrease total IgE titers in serum (Figure 8B). Notably, the dosing of IL-12 provided was sufficient to decrease HDM-driven IL-5 production locally (mLN) and reduce both IL-5 and IL-17 

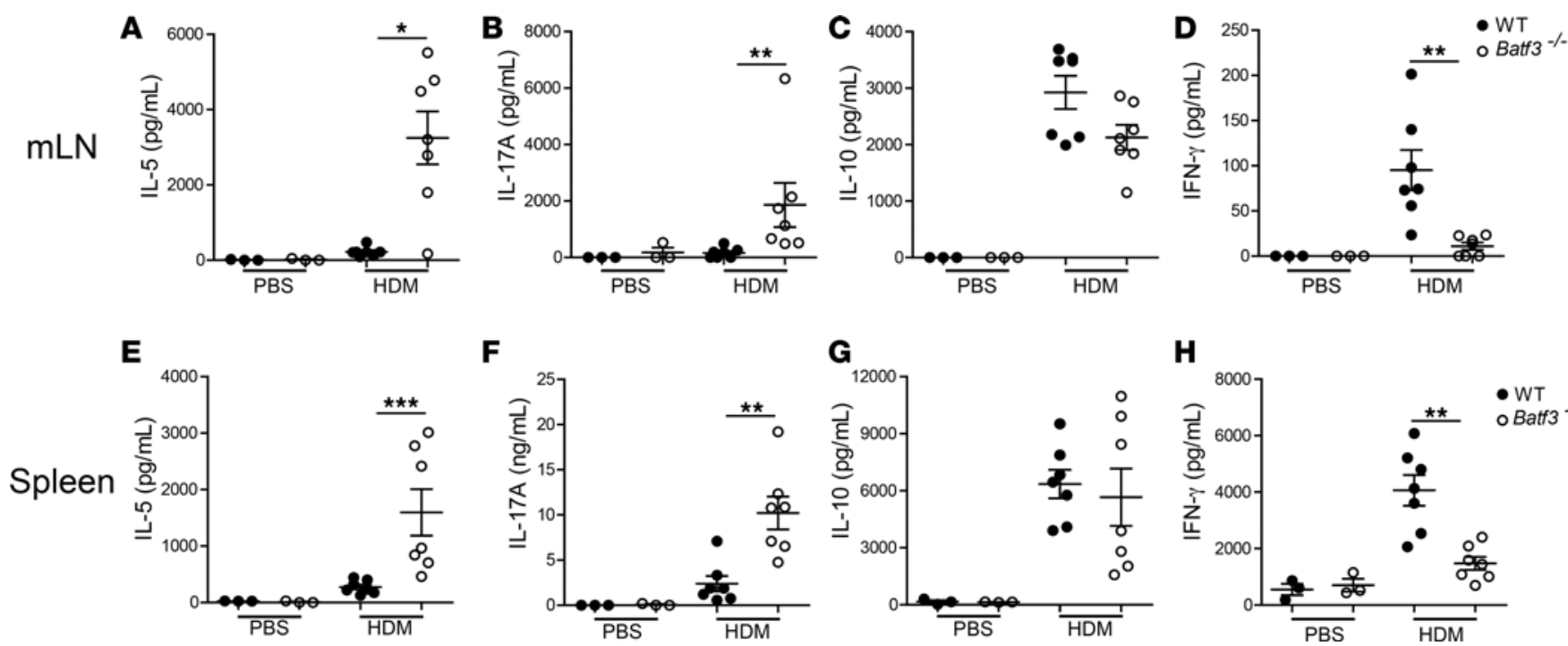

$\mathbf{F}$

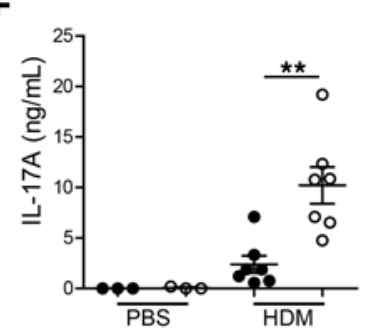

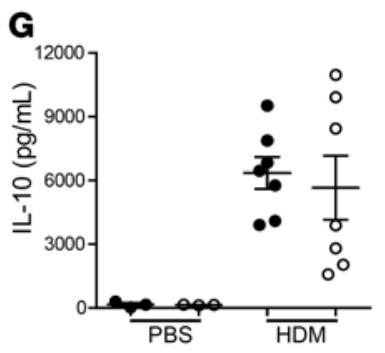

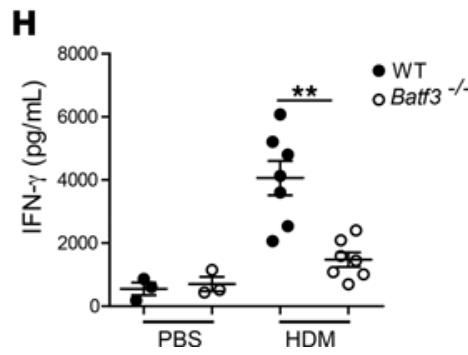

Figure 5. Enhanced Th2 and Th17 immunity upon chronic HDM challenge in Batf3 ${ }^{-/-}$mice. $\mathrm{mLNs}(\mathbf{A}-\mathbf{D})$ and splenocytes $(\mathbf{E}-\mathbf{H})$ from mice challenged over 6 weeks with $\mathrm{HDM}(3 \times 10 \mu \mathrm{g}$ doses per week) were restimulated with $\mathrm{HDM}$, and released cytokines were measured by ELISA. Individual data and mean \pm SEM from a representative independent experiment of at least 2 performed $(n=3 \mathrm{PBS}, n=7 \mathrm{HDM}) ;{ }^{*} P<0.05,{ }^{* *} P<0.01,{ }^{* * *} P<0.001$, Mann Whitney $U$ test. HDM, house dust mite; mLN, mediastinal lymph node.

production systemically (spleen) (Figure 8, C, D, F, and G). However, it was not sufficient to induce HDMdependent IFN- $\gamma$ production locally or systemically (Figure 8, E and $\mathrm{H}$ ). These results suggest that IL-12 is sufficient to control Th2 and Th17 immune responses in Batf3-deficient mice independently of the induction of Th1 immunity.

\section{Discussion}

In recent years, intense research interest has focused on the division of labor among the different pulmonary DC populations and how each contributes to the initiation and maintenance of $\mathrm{Th} 2 \mathrm{immunity}$ and allergen tolerance (9-11). The role of lung $\mathrm{CD} 103^{+} \mathrm{cDC} 1 \mathrm{~s}$ in this context is still debated and poorly understood, with reports suggesting opposing functions $(10,15-17)$. While some reports suggest that $\mathrm{CD} 103^{+} \mathrm{cDC} 1 \mathrm{~s}$ prime Th2 responses to inhaled allergens (15), others suggest that these cells are not required for Th2 cell-mediated immune responses following exposure to HDM (10). Other findings postulate that CD103+ cDC1s promote airway tolerance through the induction of FoxP $3^{+}$Tregs in models of airway inflammation (16) or through a Treg-independent production of IL-10 (17). Here, we identify Batf3-dependent CD103+ cDC1s as a key source of IL-12 upon HDM exposure; IL-12 is known to promote Th1 immunity, restraining Th2 and Th17 immunity and resulting in attenuated allergic airway inflammation.

Our findings are consistent with a previous report indicating that $\mathrm{CD} 103^{+} \mathrm{cDC} 1 \mathrm{~s}$ do not induce Th2 cell-dependent eosinophilic airway inflammation (10). However, another study suggested that pulmonary $\mathrm{CD}_{103}{ }^{+} \mathrm{cDC} 1 \mathrm{~s}$ prime Th2 responses to inhaled allergens, such as cockroach antigens and HDM (15). The authors found that $\mathrm{CD} 103^{+} \mathrm{CDC} 1 \mathrm{~s}$ prime Th1 differentiation in response to these allergens, but also found a contribution to Th2 and Th17 differentiation. The discrepancy between these findings and ours could be due to many factors, including the determination of cytokine production by coculture of DC subsets with naive CD4 T cells in the presence of anti-CD3 and anti-CD28 antibodies, contrasting with our ex vivo HDM restimulation of $\mathrm{mLNs}$ or splenocytes from sensitized and challenged mice. For in vivo studies, Nakano and colleagues used $\mathrm{Crr}^{7^{--}}$mice as a model of $\mathrm{CD} 103^{+} \mathrm{cDC} 1$ deficiency in mLNs; however, these mice also have reduced numbers of CD11b $\mathrm{b}^{+}$mig-DCs (38), which might explain their phenotype because CD11 $b^{+}$mig-DCs are major contributors to Th2 immunity and HDM-induced allergic airway inflammation (10). The authors also used BXH2/TykJ mice, which have a point mutation in the transcription factor Irf8. These mice lack CD103 ${ }^{+} \mathrm{cDC} 1 \mathrm{~s}$ in the lung and $\mathrm{CD} 8 \alpha^{+} \mathrm{cDC} 1 \mathrm{~s}$ in the spleen, but also present other immune and hematopoietic abnormalities that might explain their results (39). The different results might also reflect the effect on outcome of a different allergen dose, batch, and administration regime. 
A Gated on $\mathrm{CD} 45^{+} \mathrm{CD} 4^{+} \mathrm{T}$ cells

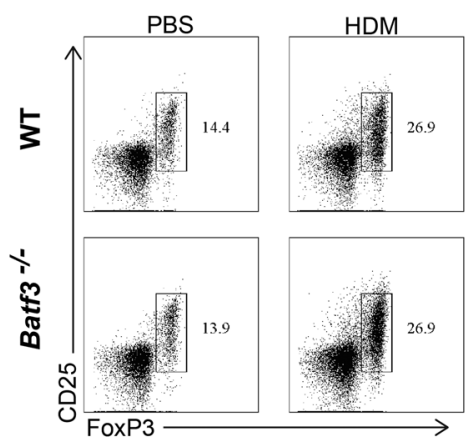

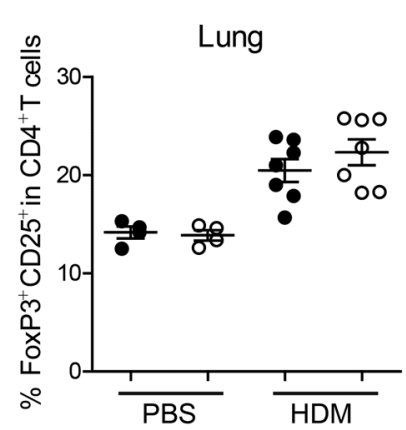

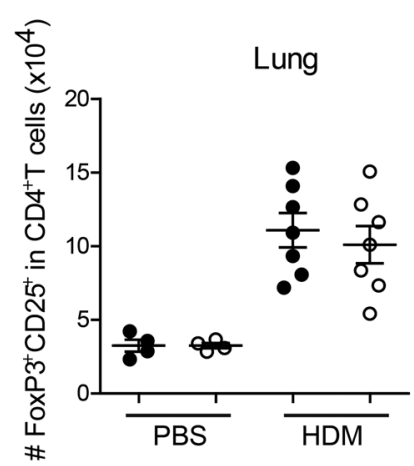

- WT

- Batf3 ${ }^{-/-}$
B

Gated on $\mathrm{CD}_{4} 5^{+} \mathrm{CD} 4^{+} \mathrm{CD}^{+} 5^{+} \mathrm{FoxP}^{+}{ }^{+} \mathrm{T}$ cells

$\frac{\infty}{\bar{\Phi}}$

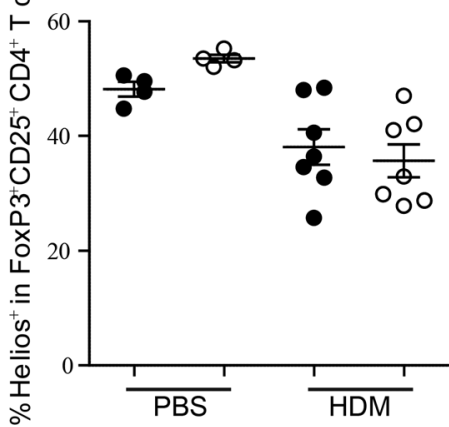

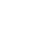

C Gated on $\mathrm{CD}_{4} 5^{+} \mathrm{CD} 4^{+} \mathrm{CD}_{2} 5^{+} \mathrm{FoxP}^{+}{ }^{+} \mathrm{T}$ cells

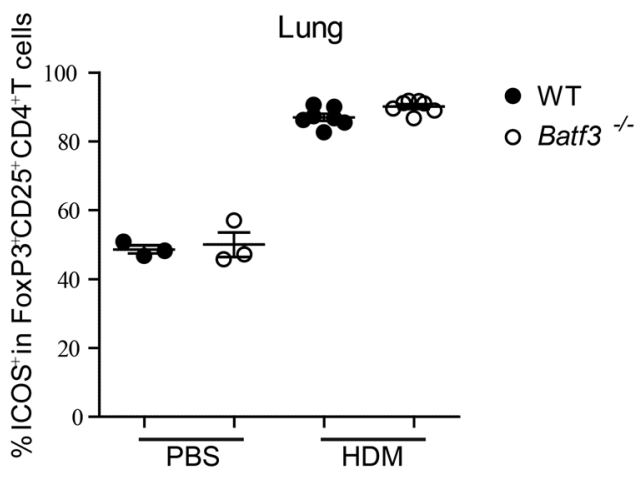

D Gated on $\mathrm{CD}_{4} 5^{+} \mathrm{CD} 4^{+} \mathrm{T}$ cells

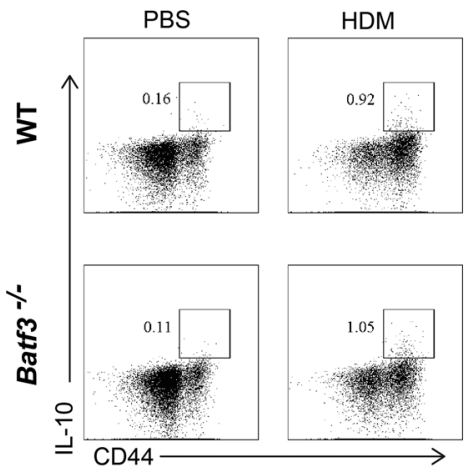
$\begin{array}{ll}- & \text { WT } \\ \circ & \text { Batf3 }\end{array}$ 
A

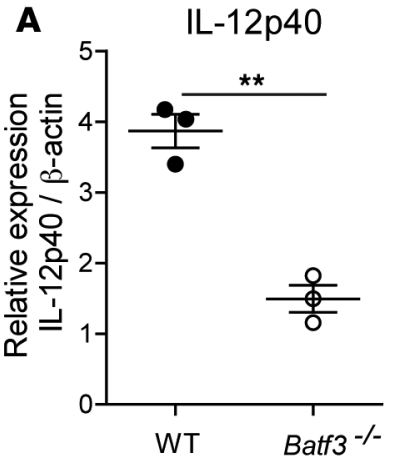

B

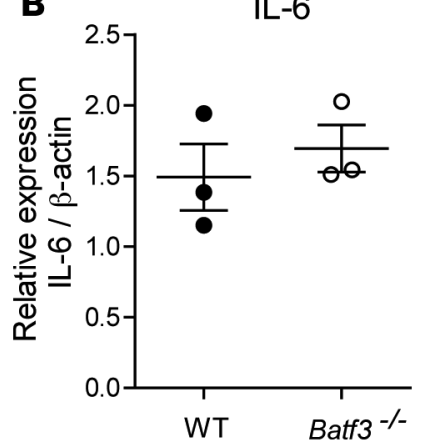

C

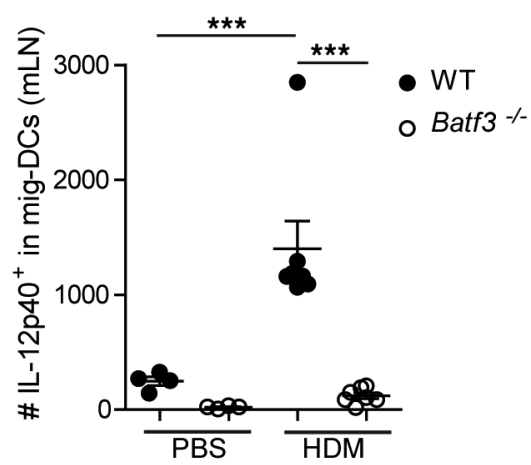

D WT Gated on $\mathrm{CD} 11 \mathrm{c}^{+} \mathrm{MHCII}^{\mathrm{hi}} \mathrm{CD} 103^{+}$mig-DCs $\mathrm{CD} 11 \mathrm{~b}^{+}$mig-DCs
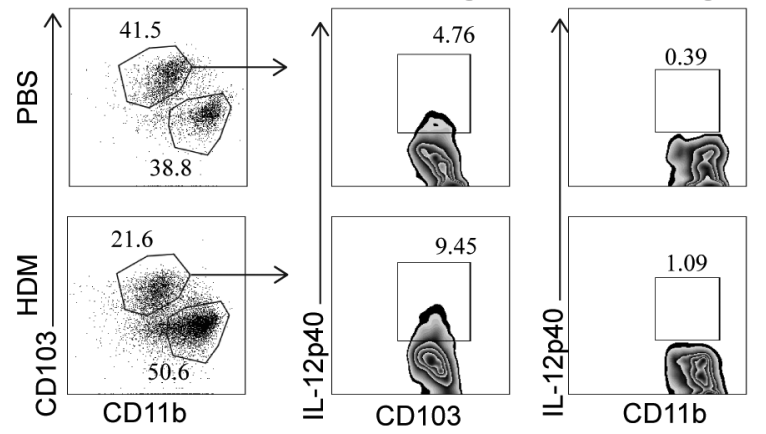

Gated on $\mathrm{CD}_{11 \mathrm{c}^{+} \mathrm{MHCl}} \mathrm{hi}^{\mathrm{CD}} 11 \mathrm{~b}^{+}$mig-DCs

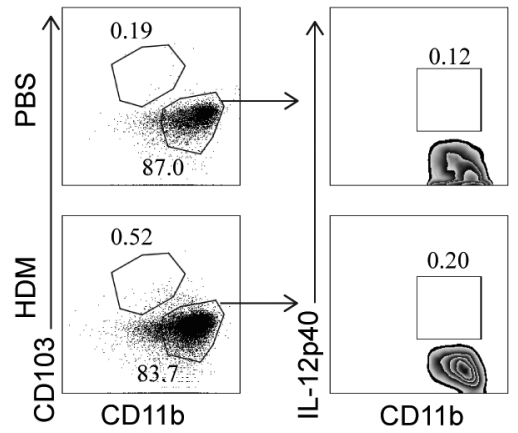

E
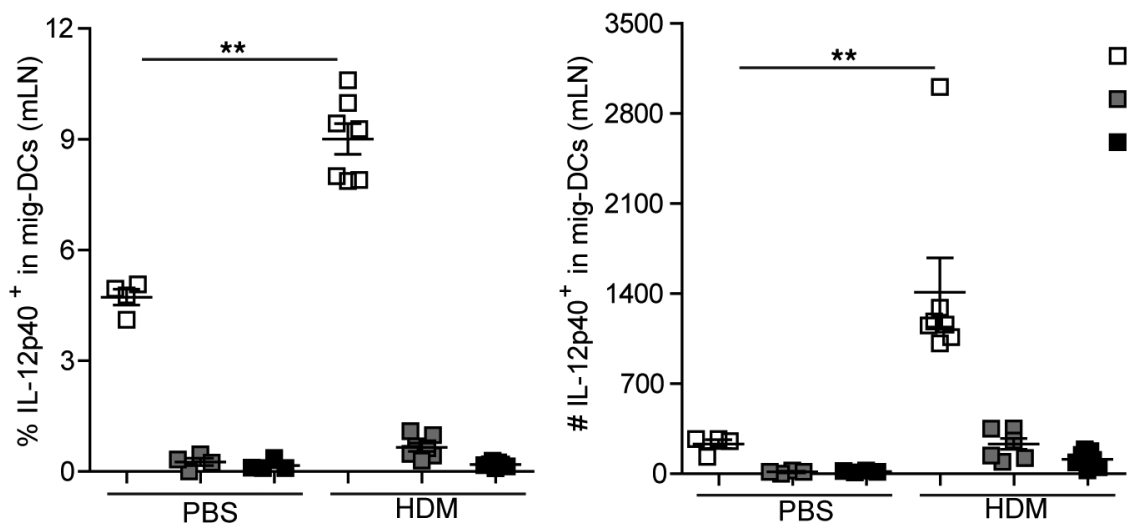

Figure 7. CD103+ mig-DCs are the main DC source of IL-12 in mLNs after HDM exposure. Mice were challenged with $100 \mu \mathrm{g}$ HDM i.n., and mLNs were collected 3 days later. (A) IL-12p40 and (B) IL-6 mRNA expression was analyzed in purified CD11c cells, and mRNA was normalized against $\beta$-actin. Data shown (mean \pm SEM) is a pool of 3 independent experiments; each symbol represents 1 experiment (5-10 mice pooled per experiment); ${ }^{* *} P<0.01$, 2-tailed Student's $t$ test. (C-E) mLN cells were stained for CD11c, MHC class II, CD103, CD11b, and intracellular IL-12p40. (C) Numbers of IL-12p40-producing cells

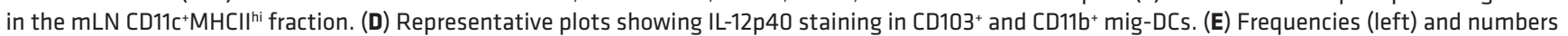
(right) of IL-12p40-producing mig-DC subsets. (C and E) Individual data and mean \pm SEM from a representative independent experiment of 3 performed ( $n$ $=4$ PBS, $n=7 \mathrm{HDM}$ ); ${ }^{* *} P<0.01,{ }^{* * *} P<0.001$, 1-way ANOVA followed by Bonferroni's post-test (C) and Mann Whitney $U$ test (A and $\left.\mathbf{E}\right)$. HDM, house dust mite; $\mathrm{mLN}$, mediastinal lymph node.

Treg induction in the lung after $\mathrm{HDM}$ exposure. Our results demonstrate that $\mathrm{CD} 103^{+} \mathrm{cDC} 1 \mathrm{~s}$ are not necessary for the expansion of FoxP3 ${ }^{+}$Tregs following i.n. sensitization and challenge with HDM. This result is consistent with findings in other organs, such as gut (19), and other settings, such as helminth infection (43). However, we cannot rule out a role for Batf3-dependent DCs in Treg generation in other settings. For instance, in models of OVA tolerance, pulmonary $\mathrm{CD}_{103^{+}} \mathrm{cDC} 1 \mathrm{~s}$ establish airway tolerance by promoting $\mathrm{T}$ cell FoxP3 expression (16). It has been proposed that $\mathrm{nTregs} \mathrm{primarily} \mathrm{target} \mathrm{autoimmune} \mathrm{responses,}$ whereas iTregs modulate the response to exogenous antigens (32). However, our model showed no evidence for a role of Batf3-dependent DCs in iTreg induction. We also examined the potential involvement of IL-10 

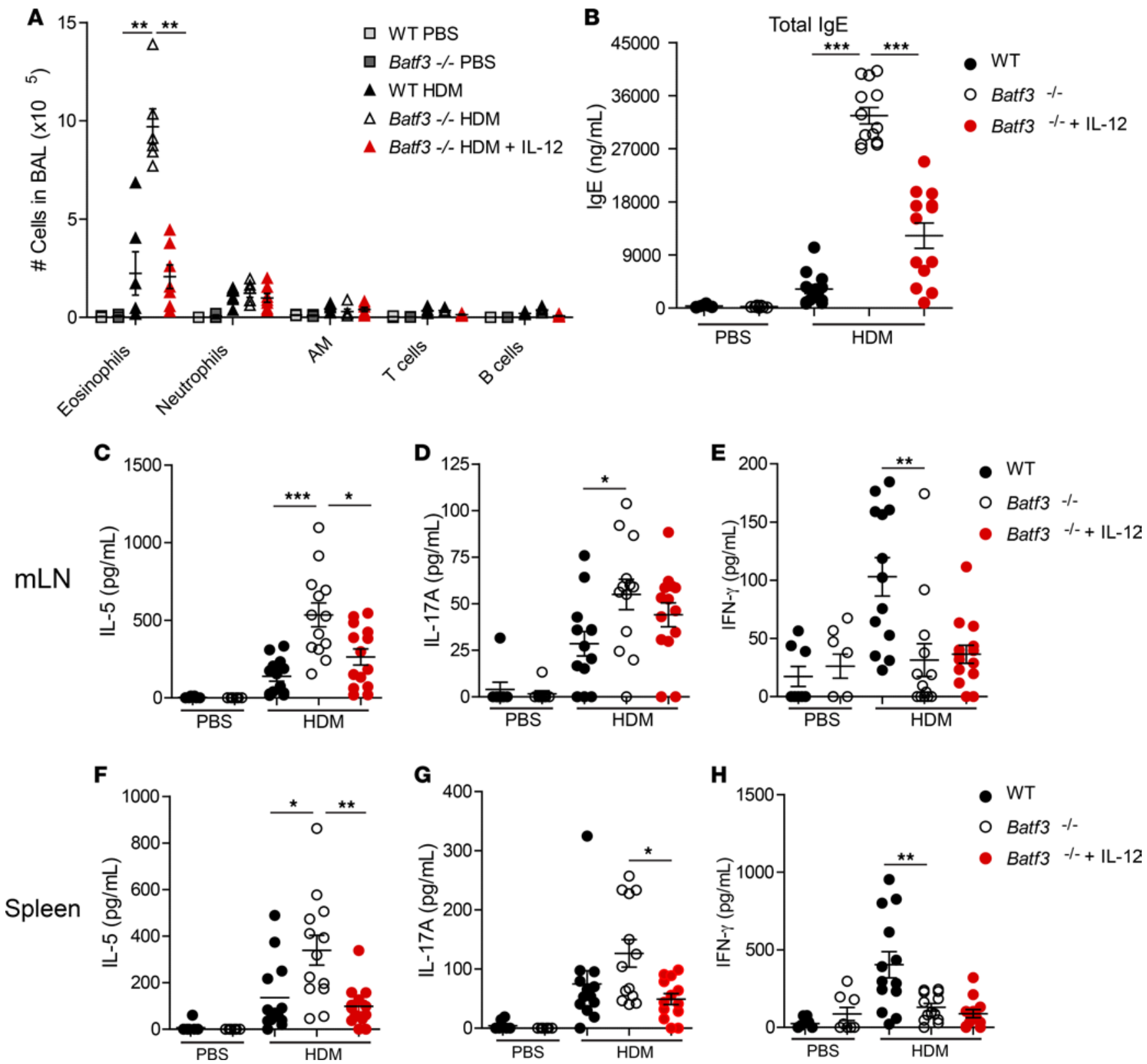

Figure 8. Intranasal administration of IL-12 dampens allergic airway inflammation in Batf3 $3^{-/-}$mice chronically exposed to $\mathrm{HDM}^{- \text {WT }}$ and $B a t f 3^{-/-}$mice were challenged for 6 weeks with HDM, as depicted in Figure 4A. Batf3 ${ }^{-/-}$mice subjected to this chronic HDM challenge were treated or not with IL-12p70 (i.n.), as indicated in Methods. (A) Inflammatory infiltrates in BAL ( $n=4$ PBS, $n=7$ HDM). (B) Total serum IgE was determined in serum by ELISA ( $n=7$ PBS $n=13 \mathrm{HDM}$ ). $\mathrm{mLNs}(\mathbf{C}-\mathbf{E})$ and splenocytes $(\mathbf{F}-\mathbf{H})$ were restimulated with HDM, and the indicated released cytokines were measured by ELISA $(n=6$ PBS, $n$ $=13$ HDM). One representative experiment $(\mathbf{A})$ or a pool of 2 independent experiments $(\mathbf{B}-\mathbf{H})$ of 3 performed is shown. Symbols denote individual mice, and lines represent mean $\pm \mathrm{SEM}$; ${ }^{*} P<0.05,{ }^{* *} P<0.01,{ }^{* * *} P<0.001$, Mann Whitney $U$ test. HDM, house dust mite; mLN, mediastinal lymph node.

because its potent immunosuppressive action is crucial not only for establishing peripheral tolerance and blocking allergic inflammation and Th17 responses, but also for protecting the host from exaggerated inflammatory responses in different contexts $(29,44,45)$. However, in our model, CD103 ${ }^{+}$cDC1 has no effect on IL-10 production by $\mathrm{CD} 4^{+} \mathrm{T}$ cells in the lung.

The expression of IL-12 by lung CD103+ mig-DCs in the steady state (46) prompted us to explore the potential involvement of this cytokine in Batf3-dependent actions during allergen-induced immune activation. Our results demonstrate that $\mathrm{HDM}$-challenged $\mathrm{CD} 103^{+} \mathrm{cDC} 1 \mathrm{~s}$ upregulate the expression and secretion of IL-12p40. This cytokine directs differentiation of naive T cells toward the Th1 effector lineage (47). Recent reports have established $\mathrm{CD} 103^{+} \mathrm{DCs}$ as a major producer of IL-12, driving protective 


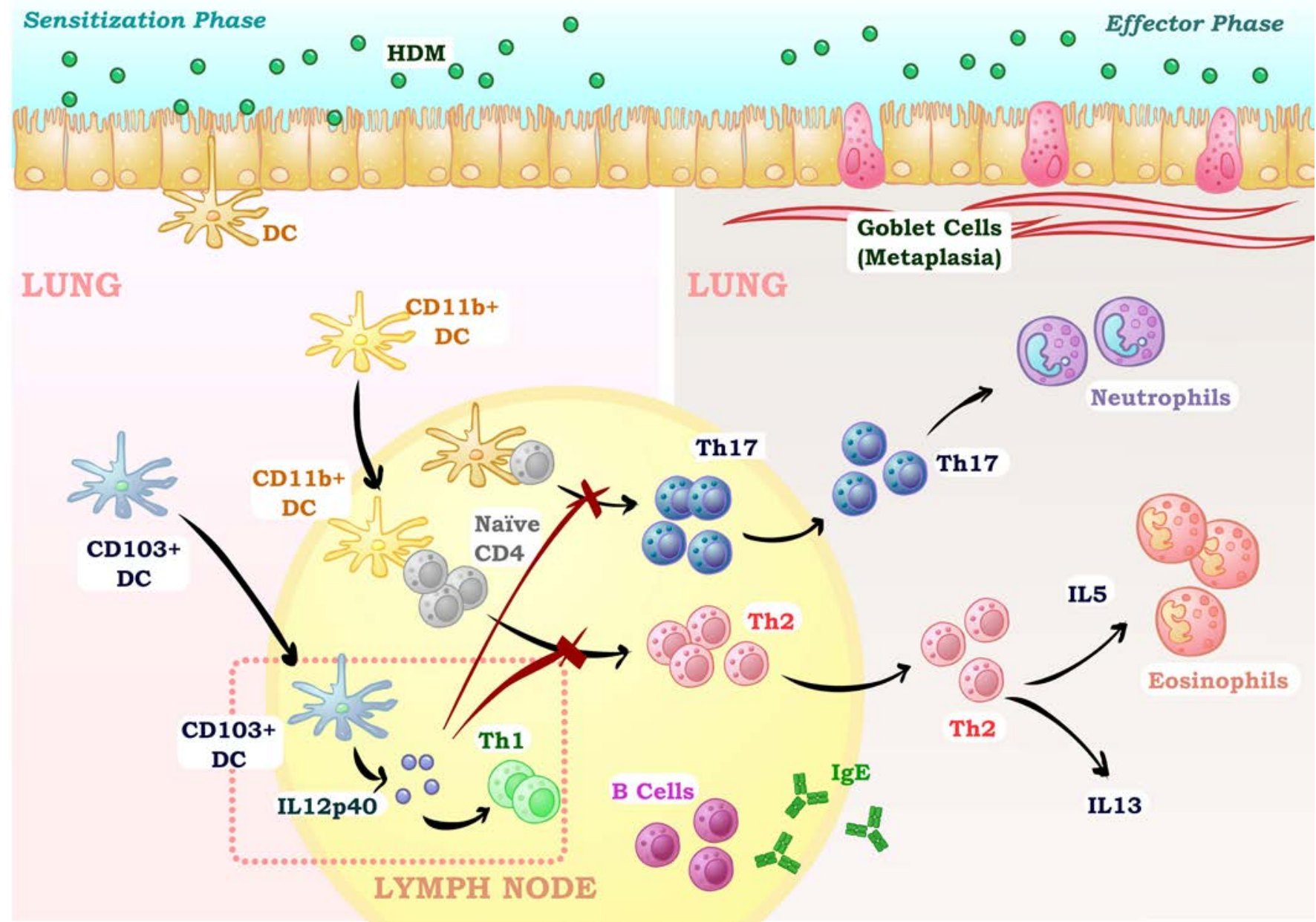

Figure 9. Proposed function of pulmonary $C D 103^{+} D C s$ in $\mathrm{HDM}$-induced asthma. Upon HDM exposure, $C D 103^{+} \mathrm{CDC1s}$ migrate from the lung to the mLNs, where they produce IL-12 that restrains Th2 and Th17 immune responses and contributes to Th1 differentiation. Batf3-dependent DCs might, thus, play a protective role in HDM-induced airway inflammation, dampening the classical features of asthma, including eosinophilia, mucus secretion, and IgE production.

Th1 immunity against several pathogens, including Toxoplasma gondii (48) and Leishmania major (49). Similarly, IL-12 production by CD103+ mig-DCs is important for suppressing helminth-driven Th2 immunity and, thus, controlling Th2-associated immunopathology during chronic Schistosoma mansoni infection (43). Our data indicate that exogenous administration of IL-12 results in restrained Th2 and Th17 immune responses, even in the absence of $\mathrm{Th} 1$ immunity, suggesting a protective role for $\mathrm{CD} 103^{+} \mathrm{cDC} 1 \mathrm{~s}$ in airway inflammation through IL-12 production and independently of the generation of Th1 responses. This is in agreement with previous reports showing that IL-12 exerts a direct inhibitory effect in Th2 and Th17 polarization in allergic airway inflammation $(50,51)$. The absence of IL-12 in Batf $3^{-1-}$ mice, thus, likely explains the skewed Th2 and Th17 immune response and the lung pathology developed following chronic HDM exposure. We do not find an increased allergic phenotype in the acute model in the absence of Batf3dependent DCs, consistent with a redundant role for these cells in triggering Th2 responses (10). However, in the long-term, absence of IL-12 produced by Batf3-dependent DCs might account for exacerbated and "unbalanced" Th2 and Th17 responses, indicating the crucial role of these cells in controlling severe chronic asthma. Interestingly, exogenous IL-12 inhibits antigen-induced airway hyperresponsiveness and other features of asthma, even when delivered during the challenge phase in sensitized mice (50). Genetically engineered DCs constitutively expressing IL-12 induce Th1 responses to inhaled antigen, preventing allergic airway inflammation; however, these DCs did not prevent eosinophilic inflammation when transferred to previously sensitized mice (52). As discussed above, different functions have been attributed to $\mathrm{CDC} 1 \mathrm{~s}$ depending on the nature of the setting/insult (including pathogens, harmless particles, and homeostasis). $\mathrm{CD}_{103^{+}} \mathrm{CDC} 1 \mathrm{~s}$ control type 1 immune response against viruses and intracellular pathogens inducing Th1 
cells. Their production of IL-12 is crucial for many of these functions (7, 43, 48, 49). However, they also induce Tregs in response to oral antigens (7) and mediate induction of Tregs in tolerized mice in an OVA model (16). This suggests that - depending on the nature of the insult, administration regime, and delivery route $-\mathrm{CD} 103^{+} \mathrm{CDC} 1 \mathrm{~s}$ can exert different functions. This is in agreement with our finding that CD103 $\mathrm{cDC} 1 \mathrm{~s}$, in the context of the clinically relevant allergen (HDM) that promotes IL-12 production in cDC1s, play a protective role in restraining Th2 and Th17 immunity and airway inflammation. Nevertheless, we cannot rule out a different function for these DCs in a different setting or in the presence of a different allergen that, for example, does not induce IL-12 production by $\mathrm{cDC} 1 \mathrm{~s}$.

Translating our results into human patients, a high heterogeneity in $\mathrm{CD} 4^{+} \mathrm{T}$ cell responses (Th1/Th2 and Th17) and dysregulated Th1/IFN- $\gamma$ immune response in severe asthma have been reported $(53,54)$. However, IL-12 administration tested in clinical trials for the treatment of asthma did not improve airway responsiveness to inhaled allergens and rather resulted in adverse effects (55). Our results indicate that IL-12 is essential for a balanced immune response in the context of asthma to HDM allergen, being crucial to control exacerbated allergic airway inflammation. Notably, our results suggest that an adequate dosing of IL-12 could result in modulation of Th2 and Th17 immunity without inducing pro-inflammatory Th1 responses. Our findings establish a role for pulmonary $\mathrm{CD} 103^{+} \mathrm{cDC} 1 \mathrm{~s}$ in the production of IL-12 and modulation of Th2 and Th17 immunity in a noninfectious setting (Figure 9). These results highlight the importance of tight regulation of the immune system in order to preserve the function and fate of the different cell populations that ultimately dictate disease outcome. Novel strategies selectively targeting CD103+ cDC1s could potentially alter the balance of allergen-induced immunity subtly and pave the way toward innovative immunotherapy for allergic airway inflammation.

\section{Methods}

Supplemental Methods are available online with this article.

Mice. Batf3 ${ }^{-1-}$ mice backcrossed more than 10 times to the C57BL/ 6 background were provided by Kenneth M. Murphy (Washington University, St. Louis, Missouri, USA) $(18,19)$ and further backcrossed with C57BL/ 6 mice at the CNIC animal facility to establish WT and Batf3 $3^{-1}$ colonies. Animals were housed and bred in specific pathogen-free conditions.

HDM extracts, asthma models, and in vivo treatment with recombinant murine IL-12. HDM (Dermatophagoides pteronyssinus) extracts (Greer Laboratories) were used throughout the experiments. For the acute model, mice were sensitized i.n. with $1 \mu \mathrm{g}$ HDM on day 1 and subsequently challenged with $10 \mu \mathrm{g}$ HDM on days 7-11 in $50 \mu 1$ PBS under light anesthesia (Figure 1A) (25). On day 3 after the last i.n. challenge (day 14 after sensitization), BAL fluid, lungs, mLNs, spleens, and serum were collected. For the chronic model, mice were i.n. challenged with $10 \mu \mathrm{g}$ HDM 3 times per week for 6 weeks, and organs were collected 3 days after the last i.n. challenge (Figure 4A). In some experiments, in parallel, Batf3 ${ }^{-/}$mice received, on the same day of HDM challenge, an i.n. administration of recombinant murine IL-12p70 (eBioscience, catalog 34-8121; $3 \times 50$ ng dose per week for 3 weeks, followed by $2 \times 75$ ng dose per week for 2 weeks). To evaluate the production of different cytokines by DCs in mLNs, mice were i.n. challenged with a high dose of HDM $(100 \mu \mathrm{g})$, and $\mathrm{mLNs}$ were harvested 3 days later.

Cell isolation and restimulation. BAL was performed by 3 consecutive flushes of the lung through the trachea with $700 \mu$ l of DMEM; cells were counted by trypan blue exclusion, and the cell profile was determined by flow cytometry. Lungs, mLNs, and spleens were collected in RPMI, and lungs and mLNs were enzyme digested. mLN cells were plated at $2 \times 10^{5}$ cells/well in 96-well round-bottom plates and restimulated for 5 days with $30 \mu \mathrm{g} / \mathrm{ml}$ HDM extract in FBS-supplemented RPMI. Similarly, splenocytes were plated at $5 \times 10^{5}$ cells/well and restimulated for 3 days. Cytokine production was measured by ELISA in culture supernatants.

Cells for flow cytometry analysis of lung infiltrates were prepared by systemic perfusion of lungs with saline. Intracellular IL-10 in lung T cells was detected after polyclonal restimulation with plated anti-CD3 $(145-2 \mathrm{C} 11,10 \mu \mathrm{g} / \mathrm{ml}$, Bio X Cell) and soluble anti-CD28 (37.51, $5 \mu \mathrm{g} / \mathrm{ml}$, Bio X Cell) in 96-well plates for 6 hours, the last 4 hours in the presence of Brefeldin A $(5 \mu \mathrm{g} / \mathrm{ml}$; Sigma-Aldrich).

Flow cytometry. Stainings were performed at $4^{\circ} \mathrm{C}$ with the appropriate antibody cocktail in cold PBS supplemented with $2.5 \%$ FBS, 2 mM EDTA and $0.1 \%$ sodium azide. Samples were processed with a BD FACSCanto flow cytometer using FACSDiva software, and data were analyzed with FlowJo software (Tree Star). CD16/CD32 (2.4G2, Tonbo Biosciences) was used to reduce nonspecific binding. To determine 
the cellular composition of BAL, cells were stained with fluorochrome-labeled antibodies directed against CD11c (HL3), Ly6G (1A8), CD11b (M1/70), Siglec-F (E50-2440), CD3 (17A2), and CD19 (1D3), purchased from BD Bioscience, and Ly6C (HK1.4) from eBioscience. For detection of Tregs and IL-10 production in the lung, cells were stained with CD45 (30-F11) purchased from BD Bioscience; CD4 (RM4-5) and CD25 (PC61.5) from Tonbo Biosciences; CD44 (IM7), FoxP3 (FJK-16s), and IL-10 (JESS-16E3) from eBioscience; and ICOS (7E.17G9) and Helios (22F6) from BioLegend. For intracellular staining, cells were fixed for 10 minutes in 4\% paraformaldehyde-PBS at room temperature, and staining was conducted for 40 minutes at $4^{\circ} \mathrm{C}$ in permeabilization buffer (1\% BSA [Sigma-Aldrich], $0.1 \%$ saponin [Sigma-Aldrich], and $0.2 \%$ sodium azide [Sigma-Aldrich]). FoxP3 and Helios in T cells were detected using the FoxP3 staining buffer set from eBioscience. For detection of cDCs in lung and $\mathrm{mLNs}$, the following fluorochrome-labeled antibodies were used: CD45 (30-F11), Siglec-F (E50-2440), I-A/I-E (MHCII, clone 2G9), CD11c (HL3), CD11b (M1/70), and CD103 (M290), from BD Biosciences, and CD8 $\alpha$ (53.6-7) from Tonbo Biosciences. Hoechst $33258(0.1 \mu \mathrm{M})$ was used as a counterstain to exclude dead cells.

For intracellular IL-12p40 staining ex vivo, mice were i.p. injected with Brefeldin A (125 $\mu \mathrm{g} / \mathrm{mouse})$ 60 hours after i.n. challenge with high-dose $\operatorname{HDM}(100 \mu \mathrm{g})$, and mLNs were collected 12 hours later $(72$ hours after challenge) in RPMI containing Brefeldin A $(5 \mu \mathrm{g} / \mathrm{ml})$. Single-cell suspensions were prepared from mLNs as described above, and cells were stained for $\mathrm{CDC}$ surface markers, fixed and permeabilized, and stained with anti-IL-12p40 mAb (C17.8) from Tonbo Biosciences.

ELISA. HDM-specific IgG1 and IgG2a and total IgE were determined in serum samples from blood collected at the end of the experiments. Briefly, for HDM-specific IgG1 and IgG2a, high binding plates were coated overnight at $4^{\circ} \mathrm{C}$ with $5 \mu \mathrm{g} / \mathrm{ml} \mathrm{HDM}$ in carbonate buffer, blocked with $10 \% \mathrm{FBS}$, and incubated with serial serum dilutions. HDM-specific immunoglobulins were detected with biotin rat anti-mouse IgG2a (R19-15) and biotin rat anti-mouse IgG1 (A85-1), both from BD Bioscience. Signal was developed by incubation with streptavidin-HRP (Sigma-Aldrich), standard TMB development (KPL), and optical density read at $450 \mathrm{~nm}$. Total serum IgE was quantified with capture mAb (purified rat anti-mouse $\operatorname{IgE}$ [R35-72]) and detection mAb (biotin-conjugated anti-mouse IgE [R35-118]) from BD Biosciences. Readings were taken from a standard curve prepared with purified mouse IgE (27-74).

Cytokine production was quantified in mLN and splenocyte culture supernatants by ELISA. IL-5, IL-10, and IFN- $\gamma$ were measured with OptEIA ELISA kits from BD Biosciences, according to the manufacturer's instructions. IL-17A production was quantified using capture mAb (rat anti-mouse IL-17A [TC1118H10]) and detection mAb (biotin-conjugated IL-17A [TC11-8H4]) both from BD Pharmingen or the mouse IL-17A ELISA Ready-Set-Go! from eBioscience. The standard curve was prepared with purified mouse IL-17 (Peprotech).

$q P C R$. Single-cell suspensions were prepared from mLNs, and cells from 5-10 mice were pooled for each biological replicate. Cell suspensions were enriched for DCs by immunomagnetic isolation using mouse anti-CD11c microbeads (N418) (Miltenyi Biotec). mRNA was extracted with the RNeasy Plus Micro kit (Qiagen, catalog 74034). RNA concentration was determined with a Nanodrop 1000 spectrophotometer (Thermo Scientific) and RNA integrity assessed with an Agilent 2100 Bioanalyzer (Caliper Life Science). Samples with RNA integrity values $>8$ were retained for further processing. RNA was reverse transcribed to cDNA using the High-Capacity cDNA Reverse Transcription Kit (Applied Biosystems). Reverse transcription PCR was carried out in a C1000 Thermal Cycler (Bio-Rad). Quantitative PCR (qPCR) was performed with the GoTaq qPCR master mix (Promega) in a 7900-FAST-384 instrument (Applied Biosystems). All reactions were performed in a $20 \mu \mathrm{l}$ volume. The housekeeping gene $\beta$-actin was used to verify equal cDNA loading. qPCR for $\beta$-actin, IL12p40, and IL6 was performed using primers from Sigma-Aldrich. Primers used for SYBR Green assays were as follows: $\beta$-actin sense, $5^{\prime}$ - GGCTGTATTCCCCTCCATCG - 3', $\beta$-actin antisense, 5' - CCAGTTGGTAACAATGCCATGT - 3'; IL-12p40 sense, 5' - GGAAGCACGGCAGCAGAATA - 3', IL-12p40 antisense, 5' - AACTTGAGGGAGAAGTAGGAATGG - 3'; IL-6 sense, 5' - CCGTGTGGTTACATCTACCCT - 3', IL-6 antisense, 5'- CGTGGTTCTGTTGATGACAGT $-3^{\prime}$. Relative cytokine expression was determined by comparison with $\beta$-actin expression.

Histology. Lungs were harvested 3 days after the final i.n. challenge with HDM and immediately fixed in $10 \%$ neutral buffered formalin. Paraffin-embedded tissues were cut into 5 - $\mu \mathrm{m}$-thick sections and stained with $H \& E$ to evaluate airway inflammation and hematoxylin-counterstained periodic acid-Schiff (PAS) to analyze mucus production. Slides were scored on a $0-5$ scale for inflammation. Sections were examined with a Leica DM 2500 light microscope and images captured with a Leica DFC 420 digital camera. 
Statistics. Statistical comparisons were made with Prism software v5 (GraphPad Software). Unless indicated otherwise in the figure legend, differences between 2 groups were calculated with the Mann-Whitney $U$ test for unpaired data, and differences between 3 or more groups were calculated with one-way ANOVA followed by Bonferroni's Multiple comparison test. Data were expressed as mean \pm SEM. Differences were considered significant at $P<0.05\left({ }^{*} P<0.05 ;{ }^{*} P<0.01 ;{ }^{*} * P<0.001\right)$.

Study approval. All animal procedures were reviewed and approved by Animal Ethics Committee at the CNIC, Madrid Autonomous University Ethics Committee, and the Community of Madrid authority. All animal procedures conformed to EU Directive 2010/63/EU and Recommendation 2007/526/EC regarding the protection of animals used for experimental and other scientific purposes, enforced in Spanish law under Real Decreto 1201/2005

\section{Author contributions}

LC and DS conceptualized the study; LC helped with methodology; LC, SCK, SMC, HMI, and PB helped with investigation; LC and DS helped with writing, supervision, and funding acquisition.

\section{Acknowledgments}

We are grateful to members of the DS lab for discussions and critical reading of the manuscript. We thank the staff at the CNIC facilities for technical support, Jesús Sánchez-Ruiz for the graphical illustration, Ruth Conde-Garrosa for technical assistance, Antonio Molina-Iracheta for histological quantification and analysis, and Simon Bartlett for editorial assistance. LC is the recipient of a European Respiratory Society Fellowship (RESPIRE2-2013-3708). Work in the DS laboratory is funded by the CNIC and grants from the Spanish Ministry of Economy, Industry and Competitiveness (MEIC) and European Fund for Regional Development (FEDER) (SAF-2013-42920-R and SAF-2016-79040-R); the European Commission (635122-PROCROP H2020); and the Fondation ACTERIA. The CNIC is supported by the MEIC and the Pro-CNIC Foundation, and is a Severo Ochoa Center of Excellence (SEV-2015-0505).

Address correspondence to: David Sancho or Laura Conejero, Centro Nacional de Investigaciones Cardiovasculares Carlos III (CNIC), Melchor Fernández Almagro, 3E-28029, Madrid, Spain. Phone: 34.662.990.4777.2010; E-mail: dsancho@cnic.es (D. Sancho); laura.conejero@cnic.es (L. Conejero).

1. Lambrecht BN, Hammad H. Lung dendritic cells in respiratory viral infection and asthma: from protection to immunopathology. Annu Rev Immunol. 2012;30:243-270.

2. Guilliams M, et al. Dendritic cells, monocytes and macrophages: a unified nomenclature based on ontogeny. Nat Rev Immunol. 2014;14(8):571-578.

3. Merad M, Sathe P, Helft J, Miller J, Mortha A. The dendritic cell lineage: ontogeny and function of dendritic cells and their subsets in the steady state and the inflamed setting. Annu Rev Immunol. 2013;31:563-604.

4. Schlitzer A, Ginhoux F. Organization of the mouse and human DC network. Curr Opin Immunol. 2014;26:90-99.

5. Kopf M, Schneider C, Nobs SP. The development and function of lung-resident macrophages and dendritic cells. Nat Immunol. 2015;16(1):36-44.

6. Guilliams M, et al. Unsupervised High-Dimensional Analysis Aligns Dendritic Cells across Tissues and Species. Immunity. 2016;45(3):669-684.

7. Durai V, Murphy KM. Functions of Murine Dendritic Cells. Immunity. 2016;45(4):719-736.

8. Furuhashi K, et al. Mouse lung CD103+ and CD11bhigh dendritic cells preferentially induce distinct CD4+ T-cell responses. Am J Respir Cell Mol Biol. 2012;46(2):165-172.

9. de Heer HJ, et al. Essential role of lung plasmacytoid dendritic cells in preventing asthmatic reactions to harmless inhaled antigen. J Exp Med. 2004;200(1):89-98.

10. Plantinga $\mathrm{M}$, et al. Conventional and monocyte-derived CD11b(+) dendritic cells initiate and maintain T helper 2 cell-mediated immunity to house dust mite allergen. Immunity. 2013;38(2):322-335.

11. Zhou Q, et al. GM-CSF-licensed CD11b+ lung dendritic cells orchestrate Th2 immunity to Blomia tropicalis. J Immunol. 2014;193(2):496-509.

12. Tussiwand R, et al. Klf4 expression in conventional dendritic cells is required for T helper 2 cell responses. Immunity. 2015;42(5):916-928.

13. Schlitzer A, et al. IRF4 transcription factor-dependent CD11b+ dendritic cells in human and mouse control mucosal IL-17 cytokine responses. Immunity. 2013;38(5):970-983.

14. Lambrecht BN, Hammad H. The immunology of asthma. Nat Immunol. 2015;16(1):45-56.

15. Nakano H, et al. Pulmonary CD103(+) dendritic cells prime Th2 responses to inhaled allergens. Mucosal Immunol. 2012;5(1):53-65.

16. Khare A, Krishnamoorthy N, Oriss TB, Fei M, Ray P, Ray A. Cutting edge: inhaled antigen upregulates retinaldehyde dehydrogenase in lung CD103+ but not plasmacytoid dendritic cells to induce Foxp3 de novo in CD4+ T cells and promote airway 
tolerance. J Immunol. 2013;191(1):25-29.

17. Engler DB, et al. Effective treatment of allergic airway inflammation with Helicobacter pylori immunomodulators requires BATF3-dependent dendritic cells and IL-10. Proc Natl Acad Sci USA. 2014;111(32):11810-11815.

18. Hildner K, et al. Batf3 deficiency reveals a critical role for CD8alpha+ dendritic cells in cytotoxic T cell immunity. Science. 2008;322(5904):1097-1100.

19. Edelson BT, et al. Peripheral CD103+ dendritic cells form a unified subset developmentally related to CD8alpha+ conventional dendritic cells. J Exp Med. 2010;207(4):823-836.

20. Seillet C, et al. CD8 $\alpha+$ DCs can be induced in the absence of transcription factors Id2, Nfil3, and Batf3. Blood. 2013;121(9):1574-1583.

21. Helft J, et al. Cross-presenting CD103+ dendritic cells are protected from influenza virus infection. J Clin Invest. 2012;122(11):4037-4047.

22. Waithman J, et al. Resident CD8(+) and migratory CD103(+) dendritic cells control CD8 T cell immunity during acute influenza infection. PLoS One. 2013;8(6):e66136.

23. Iborra S, et al. Optimal Generation of Tissue-Resident but Not Circulating Memory T Cells during Viral Infection Requires Crosspriming by DNGR-1(+) Dendritic Cells. Immunity. 2016;45(4):847-860.

24. Zelante T, et al. CD103(+) Dendritic Cells Control Th17 Cell Function in the Lung. Cell Rep. 2015;12(11):1789-1801.

25. Willart MA, et al. Interleukin-1 $\alpha$ controls allergic sensitization to inhaled house dust mite via the epithelial release of GM-CSF and IL-33. J Exp Med. 2012;209(8):1505-1517.

26. Lewkowich IP, Lajoie S, Clark JR, Herman NS, Sproles AA, Wills-Karp M. Allergen uptake, activation, and IL-23 production by pulmonary myeloid DCs drives airway hyperresponsiveness in asthma-susceptible mice. PLoS One. 2008;3(12):e3879.

27. Gurka S, Hartung E, Becker M, Kroczek RA. Mouse Conventional Dendritic Cells Can be Universally Classified Based on the Mutually Exclusive Expression of XCR1 and SIRPa. Front Immunol. 2015;6:35.

28. Tussiwand R, et al. Compensatory dendritic cell development mediated by BATF-IRF interactions. Nature. 2012;490(7421):502-507.

29. Zhao J, Lloyd CM, Noble A. Th17 responses in chronic allergic airway inflammation abrogate regulatory T-cell-mediated tolerance and contribute to airway remodeling. Mucosal Immunol. 2013;6(2):335-346.

30. Akdis M, Akdis CA. Mechanisms of allergen-specific immunotherapy: multiple suppressor factors at work in immune tolerance to allergens. J Allergy Clin Immunol. 2014;133(3):621-631.

31. Curotto de Lafaille MA, Kutchukhidze N, Shen S, Ding Y, Yee H, Lafaille JJ. Adaptive Foxp3+ regulatory T cell-dependent and -independent control of allergic inflammation. Immunity. 2008;29(1):114-126.

32. Huang H, Ma Y, Dawicki W, Zhang X, Gordon JR. Comparison of induced versus natural regulatory T cells of the same TCR specificity for induction of tolerance to an environmental antigen. J Immunol. 2013;191(3):1136-1143.

33. Thornton AM, et al. Expression of Helios, an Ikaros transcription factor family member, differentiates thymic-derived from peripherally induced Foxp3+ T regulatory cells. J Immunol. 2010;184(7):3433-3441.

34. Campbell DJ, Koch MA. Phenotypical and functional specialization of FOXP3+ regulatory T cells. Nat Rev Immunol. 2011;11(2):119-130

35. Hwang ES, Szabo SJ, Schwartzberg PL, Glimcher LH. T helper cell fate specified by kinase-mediated interaction of T-bet with GATA-3. Science. 2005;307(5708):430-433.

36. Lee YK, et al. Late developmental plasticity in the T helper 17 lineage. Immunity. 2009;30(1):92-107.

37. Laffont S, Siddiqui KR, Powrie F. Intestinal inflammation abrogates the tolerogenic properties of MLN CD103+ dendritic cells Eur J Immunol. 2010;40(7):1877-1883.

38. Nakano H, et al. Migratory properties of pulmonary dendritic cells are determined by their developmental lineage. Mucosal Immunol. 2013;6(4):678-691.

39. Turcotte K, Gauthier S, Tuite A, Mullick A, Malo D, Gros P. A mutation in the Icsbp1 gene causes susceptibility to infection and a chronic myeloid leukemia-like syndrome in BXH-2 mice. J Exp Med. 2005;201(6):881-890.

40. Bilate AM, Lafaille JJ. Induced CD4+Foxp3+ regulatory T cells in immune tolerance. Annu Rev Immunol. 2012;30:733-758.

41. Jongbloed SL, et al. Human CD141+ (BDCA-3)+ dendritic cells (DCs) represent a unique myeloid DC subset that cross-presents necrotic cell antigens. J Exp Med. 2010;207(6):1247-1260.

42. Chu CC, et al. Resident CD141 (BDCA3)+ dendritic cells in human skin produce IL-10 and induce regulatory T cells that suppress skin inflammation. J Exp Med. 2012;209(5):935-945.

43. Everts B, et al. Migratory CD103+ dendritic cells suppress helminth-driven type 2 immunity through constitutive expression of IL-12. J Exp Med. 2016;213(1):35-51.

44. Akbari O, et al. Antigen-specific regulatory T cells develop via the ICOS-ICOS-ligand pathway and inhibit allergen-induced airway hyperreactivity. Nat Med. 2002;8(9):1024-1032.

45. O'Garra A, Vieira PL, Vieira P, Goldfeld AE. IL-10-producing and naturally occurring CD4+ Tregs: limiting collateral damage. J Clin Invest. 2004;114(10):1372-1378.

46. Dalod M, Chelbi R, Malissen B, Lawrence T. Dendritic cell maturation: functional specialization through signaling specificity and transcriptional programming. EMBO J. 2014;33(10):1104-1116.

47. Macatonia SE, et al. Dendritic cells produce IL-12 and direct the development of Th1 cells from naive CD4+ T cells. J Immunol. 1995;154(10):5071-5079.

48. Mashayekhi M, et al. CD8 $\alpha(+)$ dendritic cells are the critical source of interleukin-12 that controls acute infection by Toxoplasma gondii tachyzoites. Immunity. 2011;35(2):249-259.

49. Martínez-López M, Iborra S, Conde-Garrosa R, Sancho D. Batf3-dependent CD103+ dendritic cells are major producers of IL-12 that drive local Th1 immunity against Leishmania major infection in mice. Eur J Immunol. 2015;45(1):119-129.

50. Gavett SH, O'Hearn DJ, Li X, Huang SK, Finkelman FD, Wills-Karp M. Interleukin 12 inhibits antigen-induced airway hyperresponsiveness, inflammation, and Th2 cytokine expression in mice. J Exp Med. 1995;182(5):1527-1536.

51. Durrant DM, Metzger DW. IL-12 can alleviate Th17-mediated allergic lung inflammation through induction of pulmonary IL-10 expression. Mucosal Immunol. 2010;3(3):301-311.

52. Kuipers H, et al. Dendritic cells retrovirally overexpressing IL-12 induce strong Th1 responses to inhaled antigen in the lung but 
fail to revert established Th2 sensitization. J Leukoc Biol. 2004;76(5):1028-1038.

53. Lloyd CM, Hessel EM. Functions of T cells in asthma: more than just T(H)2 cells. Nat Rev Immunol. 2010;10(12):838-848.

54. Raundhal M, et al. High IFN- $\gamma$ and low SLPI mark severe asthma in mice and humans. J Clin Invest. 2015;125(8):3037-3050.

55. Bryan SA, et al. Effects of recombinant human interleukin-12 on eosinophils, airway hyper-responsiveness, and the late asthmatic response. Lancet. 2000;356(9248):2149-2153. 